\title{
Space-Time Conservation Element and Solution Element Method and Its Applications
}

\author{
Yazhong Jiang* $*$ and Chih-Yung Wen $\ddagger$ \\ The Hong Kong Polytechnic University, Kowloon, Hong Kong, People's Republic of China \\ and \\ Deliang Zhang \\ State Key Laboratory of High Temperature Gas Dynamics, Chinese Academy of Sciences, \\ 100190 Beijing, People's Republic of China \\ https://doi.org/10.2514/1.J058928
}

\begin{abstract}
This paper reviews the development of the space-time conservation element and solution element (CESE) method and summarizes its applications in various research areas. The CESE method is a special finite-volume-type method that provides an alternative approach to numerical solutions of fluid-dynamic equations and conservation laws in various physical systems. Based on a unified treatment of time and space, this method solves the integral form of the governing equations by discretization of the space-time domain. Recent progress in CESE schemes mainly includes the construction of a family of upwind CESE schemes, the extended definitions of conservation elements and solution elements for arbitrary meshes, and a new approach to developing high-order CESE schemes. Selected applications of the CESE method (including high-speed aerodynamics, multifluid flows, detonations, and aeroacoustics) are presented. Features of the CESE method are described.
\end{abstract}

\section{Nomenclature}

\section{$\boldsymbol{B}$}

$C_{d}$

$e$

$\boldsymbol{F}$ $\boldsymbol{F}_{t}, \boldsymbol{F}_{x}, \boldsymbol{F}_{y}$

G

$\boldsymbol{h}$

$p$

$\boldsymbol{q}$

S

$T$

$t$

U

$\boldsymbol{U}_{t}, \boldsymbol{U}_{x}, \boldsymbol{U}_{y}$

$u, v$

$W$

$x, y$

$\alpha$

$\kappa$

$\mu$

$\rho$

$\rho_{g}$

$\boldsymbol{\tau}$

\section{Subscripts}

$\begin{array}{ll}i, j & =\text { index of grid points } \\ \text { inv } & =\text { inviscid } \\ L, R, C & =\text { left, right, and central } \\ \max & =\text { maximum value } \\ n & =\text { index of time levels }\end{array}$

Received 5 August 2019; revision received 14 June 2020; accepted for publication 15 June 2020; published online 7 September 2020. Copyright $($ C 2020 by the American Institute of Aeronautics and Astronautics, Inc. All rights reserved. All requests for copying and permission to reprint should be submitted to CCC at www.copyright.com; employ the eISSN 1533-385X to initiate your request. See also AIAA Rights and Permissions www.aiaa.org/ randp.

*Research Assistant, Department of Mechanical Engineering.

${ }^{\dagger}$ Professor, Interdisciplinary Division of Aeronautical and Aviation Engineering and Department of Mechanical Engineering. Associate Fellow AIAA.

Frofessor, Institute of Mechanics.

$$
\begin{array}{lll}
\text { vis } & = & \text { viscous } \\
\infty & & \text { freestream }
\end{array}
$$

Superscripts

$\begin{array}{ll}n & =\text { index of time levels } \\ -,+ & =\text { left and right } \\ * & =\text { unknowns at a new time level }\end{array}$

\section{Introduction}

$\mathbf{T}$ HE space-time conservation element and solution element (CESE) method is a special finite-volume-type method for solving equations of conservation laws. Similar to the traditional finite volume method, the CESE discretization enforces conservation. However, it uses a different procedure, which has a compact stencil, to calculate the spatial derivatives; and it achieves the same accuracy in time and space with a fully discrete one-stage formulation. Due to its attractive numerical accuracy and robustness, it has been developed into a valuable alternative method for solving problems in computational fluid dynamics (CFD). In the last two decades, the CESE method has been successfully extended and applied to a wide spectrum of physical problems, especially to nonlinear timedependent hyperbolic systems involving the dynamical evolution of waves and discontinuities.

Chang and To [1] proposed the CESE method in 1991. The principles of early CEESE schemes (referred to as $a-\varepsilon$ or $a-\alpha$ schemes, etc.) were elaborated by Chang et al. in Refs. [2-5]. To overcome the shortcoming of the $a-\alpha$ scheme, the Courant-number-insensitive (CNI) CESE scheme aimed at practical CFD problems [6-8] and became a major topic in CESE development in the early $20 \overline{0} 0 \overline{\mathrm{s}}$. This family of CESE schemes (including the $a-\varepsilon$ scheme, the $a-\alpha$ scheme, and the CNI scheme) are all based on a nondissipative core called the $a$ scheme. The main reason for such constructions is to control numerical dissipation actively or dynamically as needed. The ability to control numerical dissipation is essential in many CFD problems as well as other dispersive wave-propagation problems.

Extensions of the CESE solver to two- and three-dimensional (2-D and 3-D, respectively) unstructured meshes [9-11], 2-D and 3-D structured meshes [12], and multidimensional Navier-Stokes (NS) equations $[13,14]$ have been carried out. Efforts have also been made to design local time-stepping [15] and higher-order [16] CESE schemes. Discussions on the mathematical properties of CESE schemes have been presented by Chang [17] and Yang et al. [18]. 
Validation studies of the CESE method, along with comparative studies of this and other numerical approaches, have been conducted extensively [19-22]. In recent years, an important topic in CESE research has been the application of the CESE method to highly nonuniform and high-aspect-ratio computational meshes, which are often required in simulations of boundary layers. In particular, the CESE method has been applied to aerodynamic heating problems and direct numerical simulation of laminar or turbulent flows. It is also worth noting that CESE schemes are currently explicit in time with the associated restriction on time-step size, and extensions to implicit time-stepping CESE schemes are under investigation.

As pointed out by Zhang et al. [12], the CESE method is not an incremental improvement of a previously existing CFD method. Although physical laws are usually presented as partial differential equations (PDEs), the CESE method is entirely based on the integral form of the governing equations. By introducing 1) the concepts of the conservation element and the solution element in the discretization of the space-time domain, 2) the staggered time-marching strategy, and 3 ) the direct storage and updating of the derivatives of physical quantities, the CESE method possesses a complete conservation property and a high degree of locality. Results of numerical experiments indicate that the simplest CESE scheme has a second-order convergence rate, whereas its ability to capture discontinuities is desirable in many applications [23]. Owing to its accuracy and robustness, the CESE method is employed in the CFD module of the simulation software LS-DYNA [24].

This paper provides an overview of the CESE method and its applications in different fields, with an emphasis on recent works. After a presentation of the fundamentals of CESE in Secs. II and III, developments of the CESE method over the past five years $\overline{\text { are }}$ highlighted in Sec. IV. For the early history of CESE, one can refer to the excellent reviews by Liu et al. [23] and Wang [25]. Section $\underline{\mathrm{V}}$ provides remarks on some numerical features of the CESE schemes. In Sec. VI, a comprehensive survey of applications of the CESE method is presented, with a broad scope covering aerodynamics, multifluid flows, detonations, aeroacoustics, and many other research areas. Finally, conclusions are given in Sec. VII.

\section{Space-Time Integral Form of Governing Equations}

Governing equations for a specific physical problem can be formulated in different ways. However, the form of equations that is used in a numerical method will have a fundamental influence on its numerical performance. A major feature of the CESE method is the adoption of a space-time integral form of the governing equations, in which time and space are treated on the same footing [1]. For illustrative purposes, we consider the Navier-Stokes equations for two-dimensional compressible flows as

$$
\frac{\partial \boldsymbol{U}}{\partial t}+\frac{\partial \boldsymbol{F}_{\mathrm{inv}}}{\partial x}+\frac{\partial \boldsymbol{G}_{\mathrm{inv}}}{\partial y}=\frac{\partial \boldsymbol{F}_{\mathrm{vis}}}{\partial x}+\frac{\partial \boldsymbol{G}_{\mathrm{vis}}}{\partial y}
$$

where $\boldsymbol{U}$ denotes the vector of conservative variables such that

$$
\boldsymbol{U}=\left(\begin{array}{c}
\rho \\
\rho u \\
\rho v \\
\rho e
\end{array}\right)
$$

where $\boldsymbol{F}_{\text {inv }}$ and $\boldsymbol{G}_{\text {inv }}$ are the inviscid fluxes of the form

$$
\boldsymbol{F}_{\mathrm{inv}}=\left(\begin{array}{c}
\rho u \\
\rho u^{2}+p \\
\rho u v \\
(\rho e+p) u
\end{array}\right), \quad \boldsymbol{G}_{\mathrm{inv}}=\left(\begin{array}{c}
\rho v \\
\rho u v \\
\rho v^{2}+p \\
(\rho e+p) v
\end{array}\right)
$$

and $\boldsymbol{F}_{\text {vis }}$ and $\boldsymbol{G}_{\text {vis }}$ are the viscous fluxes, which can be written as

$$
\boldsymbol{F}_{\mathrm{vis}}=\left(\begin{array}{c}
0 \\
\tau_{x x} \\
\tau_{x y} \\
\tau_{x x} u+\tau_{x y} v-q_{x}
\end{array}\right), \boldsymbol{G}_{\mathrm{vis}}=\left(\begin{array}{c}
0 \\
\tau_{x y} \\
\tau_{y y} \\
\tau_{x y} u+\tau_{y y} v-q_{y}
\end{array}\right)
$$

In Eqs. (2) and (3), $\rho$ is the density of the fluid; $u$ and $v$ are the $x$ and $y$ components of the flow velocity, respectively; $p$ is the static pressure; and $e$ is the total energy per unit mass of the fluid. In Eq. (4), $\boldsymbol{\tau}$ is the viscous-stress tensor and $\boldsymbol{q}$ is the heat-flux vector. By using Newton's formula for viscous stress and Stokes's hypothesis, $\tau$ can be written as

$$
\boldsymbol{\tau}=\mu\left((\nabla \boldsymbol{u})+(\nabla \boldsymbol{u})^{T}-\frac{2}{3}(\nabla \cdot \boldsymbol{u}) \boldsymbol{I}\right)
$$

where $\mu$ is the viscosity, $\boldsymbol{u}$ is the flow velocity vector, $\boldsymbol{I}$ is the unit tensor, and $\nabla$ is the gradient operator. The heat-flux vector $\boldsymbol{q}$ can be expressed by Fourier's law of heat conduction

$$
q=-\kappa \nabla T
$$

where $\kappa$ is the thermal conductivity, and $T$ is the temperature. Note that once the transport properties and the equation of state are provided, Eq. (1) becomes a closed set of equations.

To rewrite Eq. (1) in a more compact form, we introduce the notation

$$
\boldsymbol{F}=\boldsymbol{F}_{\mathrm{inv}}-\boldsymbol{F}_{\mathrm{vis}}, \quad \boldsymbol{G}=\boldsymbol{G}_{\mathrm{inv}}-\boldsymbol{G}_{\mathrm{vis}}
$$

Next, with a unified treatment of time and space, we define $x, y$, and $t$ as coordinates in a three-dimensional Euclidean space $E^{3}$. Hence, Eq. (1) can be written in a divergence-free form as

$$
\nabla \cdot \boldsymbol{h}=0, \quad \boldsymbol{h}=(\boldsymbol{F}, \boldsymbol{G}, \boldsymbol{U})
$$

After applying Gauss's divergence theorem to an arbitrary control volume $V$ in $E^{3}$, of which the boundary surface is denoted by $S(V)$, we finally write the governing equations as

$$
\oint_{S(V)} \boldsymbol{h} \cdot \boldsymbol{n} \mathrm{d} S=0
$$

where $\boldsymbol{n}$ denotes the unit outward normal vector on $S(V)$. Clearly, this equation gives a direct description of the space-time conservation of mass, momentum, and energy in fluid flows; and it faithfully preserves the original physical laws. In the CESE method, this integral form [Eq. (9)] serves as the starting point for the construction of numerical schemes.

\section{Conservation Element and Solution Element Method}

This section is devoted to demonstrating the basic ideas of the CESE method. For clarity of mathematical notation without loss of generality, the CESE method is applied to a one-dimensional (1-D) scalar model equation of the form

$$
\frac{\partial u}{\partial t}+\frac{\partial f(u)}{\partial x}=0
$$

Numerical discretization and solution schemes for Eq. (10) will be presented in this section.

\section{A. Discretization}

The first process of the CESE method is to discretize the space-time domain that is relevant to the computation. The discretization procedure begins with generating the mesh for the physical space and determining the time-step size for the time-marching algorithm, which is similar to most CFD methods. The features of the CESE 
discretization include the arrangement of the solution points, the selection of the unknown variables to be calculated and stored at each solution point, and the construction of control volumes for the space-time integral form of the governing equation. All these features can be illustrated by introducing two special concepts: the conservation element (CE) and the solution element (SE).

Consider the application of the CESE method to Eq. (10). With a uniform division of the 1-D physical space and a constant time step, the 2-D $x$ - $t$ plane is discretized by a space-time mesh as shown in Fig. 1 (solid lines). The spatial coordinate of the $j$ th mesh node is denoted by $x_{j}$. The position of each cell center is $x_{j+1 / 2}=\left(x_{j}+x_{j+1}\right) / 2$, and the cell size is $x_{j+1}-x_{j}=\Delta x$. In this time-marching algorithm, each step $\left[t_{n-1}, t_{n}\right]$ consists of two half-steps: $\left[t_{n-1}, t_{n-1 / 2}\right]$ and $\left[t_{n-1 / 2}, t_{n}\right]$. The time-step size is defined as $\Delta t=t_{n}-t_{n-1}$.

The unknown function $u(x, t)$ is represented by the discrete values of $u$ at a set of specific space-time points, called solution points. For integer time levels $\left\{t_{0}, t_{1}, \ldots, t_{n} \ldots\right\}$, the solution points are mesh nodes (circles in Fig. 1). For half-integer time levels $\left\{t_{1 / 2}, t_{3 / 2}, \ldots, t_{n+1 / 2} \ldots\right\}$, the solution points are cell centers (squares in Fig. 1). In other words, a staggered mesh is used at each intermediate time level. In the basic CESE scheme, unknown variables that need to be calculated and stored at each solution point [e.g., point $(j, n)]$ include not only $u$ but also its spatial derivative $u_{x}$ :

$$
u_{j}^{n} \equiv u\left(x_{j}, t_{n}\right), \quad\left(u_{x}\right)_{j}^{n} \equiv \frac{\partial u}{\partial x}\left(x_{j}, t_{n}\right)
$$

A schematic of the paths of information flow in a single CESE time step is shown in Fig. 2. As seen in this figure, the CESE scheme results in a highly compact stencil in space and time. If a half-step is treated as the basic iteration, the half-width of the symmetric stencil is $\Delta x / 2$ because unknowns at point $(j, n)$ only depend on the data stored at $(j-1 / 2, n-1 / 2)$ and $(j+1 / 2, n-1 / 2)$.

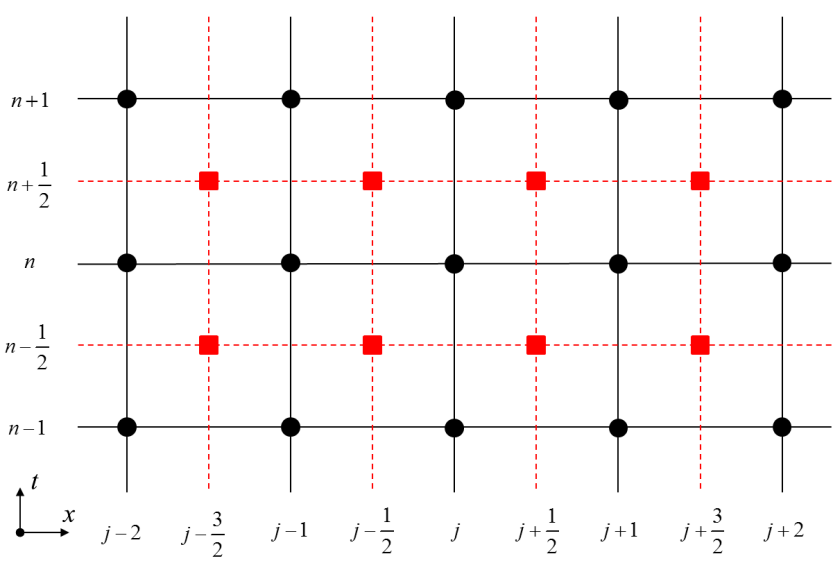

Fig. 1 Computational mesh and the arrangement of solution points.

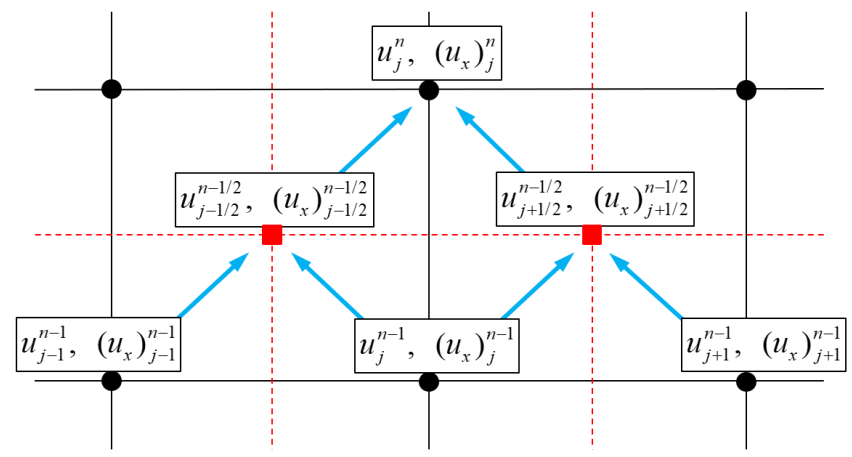

Fig. 2 Time-marching variables and the information flow in one CESE time step.
Based on the space-time mesh shown in Fig. 1, a set of small space-time elements (named conservation elements) can be constructed as demonstrated in Fig. 3 . To each solution point, a CE is assigned. For example, the $\mathrm{CE}$ for point $(j, n)$, denoted as $(\mathrm{CE})_{j}^{n}$, is the rectangle whose four vertices are the points $(j-1 / 2, n-1 / 2)$, $(j+1 / 2, n-1 / 2),(j+1 / 2, n)$, and $(j-1 / 2, n)$. Clearly, the CEs cover the whole space-time domain without overlap. It is worth noting that the arrangement of CEs in two successive half-time steps is staggered. Within each conservation element, the space-time integral form of Eq. (10) is numerically implemented, and discrete equations for unknowns are established.

When performing the space-time integration of Eq. (10) over a CE, an important question is how to evaluate $u$ and $f$ along the boundary of the CE. This leads to the introduction of a solution element for each solution point. The definition of $(\mathrm{SE})_{j}^{n}$ is shown in Fig. $\underline{4}$, where two line segments bisect each other at point $(j, n)$, forming a cross with four endpoints of $(j+1 / 2, n),(j, n+1 / 2),(j-1 / 2, n)$, and $(j, n-1 / 2)$. In the same way, SEs for half-integer points can be defined. Note that each conservation element $(\mathrm{CE})_{j}^{n}$ is bounded by three solution elements: $(\mathrm{SE})_{j}^{n},(\mathrm{SE})_{j-1 / 2}^{n-1 / 2}$, and (SE) ${ }_{j+1 / 2}^{n-1 / 2}$. The SEs cover the boundaries of every $\mathrm{CE}$ with no overlap. Inside each $\mathrm{SE}$, the functions $u(x, t)$ and $f(x, t)$ are assumed to be linear and can be approximated by first-order Taylor expansions about the center of the SE. To be specific, in the solution element $(\mathrm{SE})_{j}^{n}, u$ and $f$ are constructed as

$$
u(x, t)=u_{j}^{n}+\left(u_{x}\right)_{j}^{n}\left(x-x_{j}\right)+\left(u_{t}\right)_{j}^{n}\left(t-t_{n}\right),(x, t) \in(\mathrm{SE})_{j}^{n}
$$

and

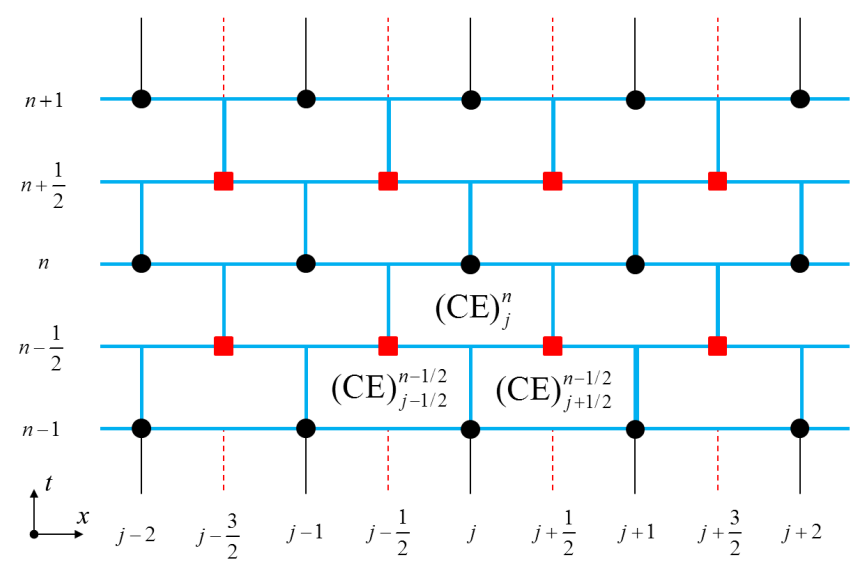

Fig. 3 Definition and arrangement of conservation elements.

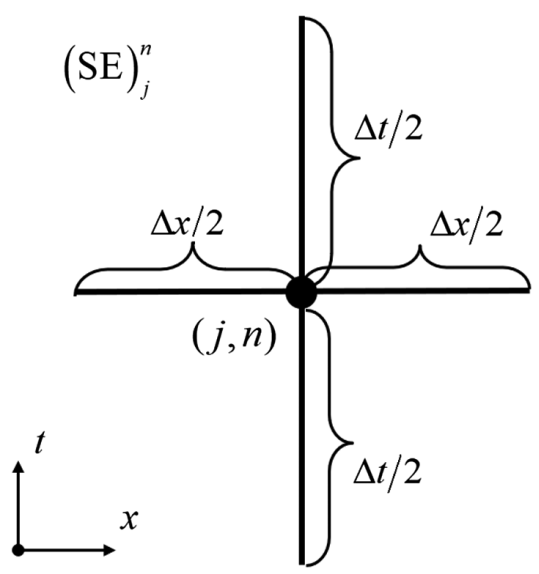

Fig. 4 Definition of the solution element associated with solution point $(j, n)$. 


$$
f(x, t)=f_{j}^{n}+\left(f_{x}\right)_{j}^{n}\left(x-x_{j}\right)+\left(f_{t}\right)_{j}^{n}\left(t-t_{n}\right),(x, t) \in(\mathrm{SE})_{j}^{n}
$$

where $u_{t}$ and $f_{t}$ are the temporal derivatives of $u$ and $f$, respectively.

\section{B. Nondissipative Core Scheme}

In this section, a nondissipative CESE scheme, named the $a$ scheme, is established to solve the 1-D scalar conservation law Eq. (10). A space-time flux vector is defined as

$$
\boldsymbol{h}=(f, u)
$$

where $f$ and $u$ can be regarded as the components of flux vector $\boldsymbol{h}$ in the $x$ direction and $t$ direction, respectively. With this definition, Eq. (10) can be converted into the space-time integral form of Eq. (9) by following the procedure in Sec. II.

Consider a half-step marching from time level $n-1 / 2$ to time level $n$. At the solution point $(j, n)$, two independent unknowns $\left[u_{j}^{n}\right.$ and $\left(u_{x}\right)_{j}^{n}$ ] need to be calculated simultaneously. Thus, two algebraic equations need to be formulated by discretizing the integral conservation law. For this purpose, we split the conservation element $(\mathrm{CE})_{j}^{n}$ into two subelements: $\left(\mathrm{CE}^{-}\right)_{j}^{n}$ and $\left(\mathrm{CE}^{+}\right)_{j}^{n}$. As shown in Fig. $\underline{5}$, $\left(\mathrm{CE}^{-}\right)_{j}^{n}$ is the rectangle $A C D E$, and $\left(\mathrm{CE}^{+}\right)_{j}^{n}$ is the rectangle $C B F D$. Each edge of these rectangles belongs to one of the three SEs associated with $(\mathrm{CE})_{j}^{n}$.

Next, the space-time integral conservation law is implemented over each of the sub-CEs. Let the control volume $V$ in Eq. (9) be $\left(\mathrm{CE}^{-}\right)_{j}^{n}$ and $\left(\mathrm{CE}^{+}\right)_{j}^{n}$ in turn, and so one can obtain

$$
\begin{aligned}
& \oint_{S\left(\mathrm{CE}^{-}\right)_{j}^{n}} \boldsymbol{h} \cdot \boldsymbol{n} \mathrm{d} S=0 \\
& \oint_{S\left(\mathrm{CE}^{+}\right)_{j}^{n}} \boldsymbol{h} \cdot \boldsymbol{n} \mathrm{d} S=0
\end{aligned}
$$

where $\boldsymbol{h}$ is expressed by Eq. (14), and $\boldsymbol{n}$ is the unit outward normal vector on the boundary of $\left(\mathrm{CE}^{-}\right)_{j}^{n}$ or $\left(\mathrm{CE}^{+}\right)_{j}^{n}$.

As marked in Fig. 6, the average values of $u(x, t)$ on line segments $D E, D F, A C$, and $B \bar{C}$ are denoted by $U_{L}^{*}, U_{R}^{*}, U_{L}$, and $U_{R}$, respectively. In addition, $F_{L}, F_{R}$, and $F_{C}$ represent the average values of $f(x, t)$ on line segments $A E, B F$, and $C D$ (note that $C D$ is the interface between two sub-CEs), respectively. With this notation, integral equations (15) and (16) can be expressed as

$$
\begin{aligned}
& U_{L}^{*} \frac{\Delta x}{2}=U_{L} \frac{\Delta x}{2}+\left(F_{L}-F_{C}\right) \frac{\Delta t}{2} \\
& U_{R}^{*} \frac{\Delta x}{2}=U_{R} \frac{\Delta x}{2}+\left(F_{C}-F_{R}\right) \frac{\Delta t}{2}
\end{aligned}
$$

which explicitly state the balance of space-time flux in each sub-CE.

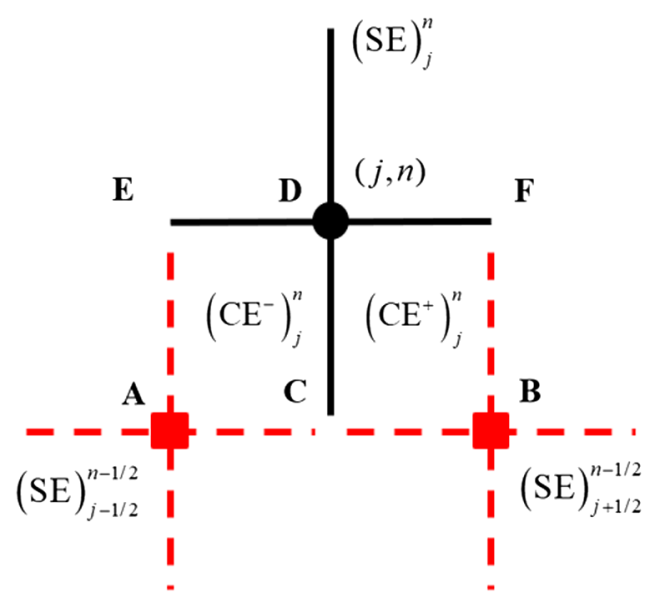

Fig. 5 Definition of sub-CEs: $\mathrm{CE}^{-}$and $\mathrm{CE}^{+}$.
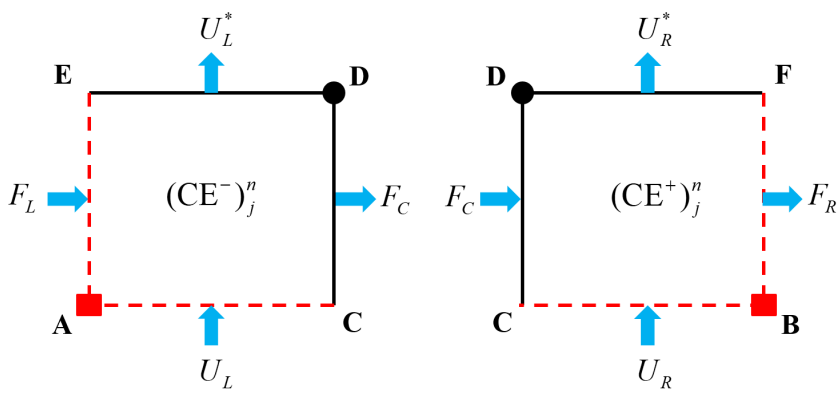

Fig. 6 Space-time flux through the boundaries of sub-CEs.

To proceed with the scheme construction, $U_{L}^{*}, U_{R}^{*}, U_{L}, U_{R}, F_{L}$, $F_{R}$, and $F_{C}$ in Eqs. (17) and (18) must be related to the time-marching variables (i.e., $u$ and $\bar{u}_{x}$ ) at the solution points using the concept of the solution element. Recall that $u$ and $f$ are assumed to be linear functions of $x$ and $t$ inside each individual SE and can be calculated by first-order Taylor expansions about the solution point. Since line segments $A C$ and $A E$ belong to (SE) ${ }_{j-1 / 2}^{n-1 / 2}, U_{L}$ and $F_{L}$ can actually be expressed in terms of the known information stored at $(j-1 / 2$, $n-1 / 2$ ), namely, point $A$ in Figs. $\underline{5}$ and $\underline{6}$. Performing Taylor expansions in $(\mathrm{SE})_{j-1 / 2}^{n-1 / 2}$ yields

$$
\begin{aligned}
U_{L} & =u_{j-1 / 2}^{n-1 / 2}+\frac{\Delta x}{4}\left(u_{x}\right)_{j-1 / 2}^{n-1 / 2} \\
F_{L} & =f_{j-1 / 2}^{n-1 / 2}+\frac{\Delta t}{4}\left(f_{t}\right)_{j-1 / 2}^{n-1 / 2}
\end{aligned}
$$

Similarly, since $B C$ and $B F$ belong to (SE) ${ }_{j+1 / 2}^{n-1 / 2}, U_{R}$ and $F_{R}$ can be evaluated by the Taylor expansions about solution point $(j+1 / 2$, $n-1 / 2)$ :

$$
\begin{aligned}
& U_{R}=u_{j+1 / 2}^{n-1 / 2}-\frac{\Delta x}{4}\left(u_{x}\right)_{j+1 / 2}^{n-1 / 2} \\
& F_{R}=f_{j+1 / 2}^{n-1 / 2}+\frac{\Delta t}{4}\left(f_{t}\right)_{j+1 / 2}^{n-1 / 2}
\end{aligned}
$$

In Eqs. (19-22), $u$ and $u_{x}$ at time level $n-1 / 2$ are already known. Since $f=\bar{f}(u)$ is a prescribed function in the conservation law, one has

$$
f_{j \pm 1 / 2}^{n-1 / 2}=f\left(u_{j \pm 1 / 2}^{n-1 / 2}\right)
$$

By using the chain rule, the spatial derivative of $f$ is expressed as

$$
\left(f_{x}\right)_{j \pm 1 / 2}^{n-1 / 2}=\left[(\partial f / \partial u) u_{x}\right]_{j \pm 1 / 2}^{n-1 / 2}
$$

The temporal derivative of $u$ can be obtained using Eq. (10), which is the governing equation itself:

$$
\left(u_{t}\right)_{j \pm 1 / 2}^{n-1 / 2}=-\left(f_{x}\right)_{j \pm 1 / 2}^{n-1 / 2}
$$

By using the chain rule again, the temporal derivative of $f$ is derived as

$$
\left(f_{t}\right)_{j \pm 1 / 2}^{n-1 / 2}=\left[(\partial f / \partial u) u_{t}\right]_{j \pm 1 / 2}^{n-1 / 2}
$$

Hence $U_{L}, F_{L}, U_{R}$, and $F_{R}$ can be explicitly calculated using the preceding formulas.

Next, consider unknowns at time level $n$. The first-order Taylor expansion in (SE) ${ }_{j}^{n}$ relates $U_{L}^{*}$ and $U_{R}^{*}$ to unknowns $u_{j}^{n}$ and $\left(u_{x}\right)_{j}^{n}$ at time level $n$ : 


$$
\begin{aligned}
& U_{L}^{*}=u_{j}^{n}-\frac{\Delta x}{4}\left(u_{x}\right)_{j}^{n} \\
& U_{R}^{*}=u_{j}^{n}+\frac{\Delta x}{4}\left(u_{x}\right)_{j}^{n}
\end{aligned}
$$

Substituting Eqs. (27) and (28) into Eqs. (17) and (18), one obtains

$$
\begin{aligned}
& u_{j}^{n}-\frac{\Delta x}{4}\left(u_{x}\right)_{j}^{n}=U_{L}+\left(F_{L}-F_{C}\right) \frac{\Delta t}{\Delta x} \\
& u_{j}^{n}+\frac{\Delta x}{4}\left(u_{x}\right)_{j}^{n}=U_{R}+\left(F_{C}-F_{R}\right) \frac{\Delta t}{\Delta x}
\end{aligned}
$$

From the sum of Eqs. (29) and (30), one can derive

$$
u_{j}^{n}=\frac{1}{2}\left(U_{L}+U_{R}\right)+\frac{\Delta t}{2 \Delta x}\left(F_{L}-F_{R}\right)
$$

and from the difference of Eqs. (29) and (30), one can derive

$$
\frac{\Delta x}{4}\left(u_{x}\right)_{j}^{n}=\frac{1}{2}\left(U_{R}-U_{L}\right)+\frac{\Delta t}{2 \Delta x}\left(2 F_{C}-F_{L}-F_{R}\right)
$$

So far, Eq. (31) is an explicit time-marching formula for $u_{j}^{n}$, but $\left(u_{x}\right)_{j}^{n}$ cannot be directly provided by Eq. (32). This is because $F_{C}$, which denotes the average value of flux $f$ through the interface $C D$, has not been addressed. In the original CESE scheme, $F_{C}$ is given by the Taylor expansion in $(\mathrm{SE})_{j}^{n}$, i.e.,

$$
F_{C}=f_{j}^{n}-\frac{\Delta t}{4}\left(f_{t}\right)_{j}^{n}
$$

With a procedure analogous to Eqs. $(\underline{23}-\underline{26}), f_{j}^{n}$ and $\left(f_{t}\right)_{j}^{n}$ can be expressed in terms of $u_{j}^{n}$ and $\left(u_{x}\right)_{j}^{n}$. Since $u_{j}^{n}$ is determined by Eq. (31), the only unknown in Eq. (32) is $\left(u_{x}\right)_{j}^{n}$, and a time-marching formula for $\left(u_{x}\right)_{j}^{n}$ can eventually be derived.

Note that a complete CESE time step consists of two half-steps: a half-step marching from nodes to centers and a half-step marching from centers to nodes. The marching schemes for both half-steps are identical, except for the indexes of the solution points. At the initial time (usually $t_{0}=0$ ), the initial conditions of $u$ and $u_{x}$ must be specified. At the boundaries of $x$, corresponding boundary conditions for $u$ and $u_{x}$ need to be implemented.

The aforementioned CESE scheme is referred to as the $a$ scheme in the literature [2]. A remarkable feature of the $a$ scheme is its use of Taylor expansion in the inverse-time direction to relate the interface flux $F_{C}$ to the marching variables [see Eq. (33)]. Such a treatment results in the space-time inversion invariance of the $a$ scheme, which further makes this scheme nondissipative [17]. From a historical perspective, the $a$ scheme serves as the nondissipative core of subsequent CESE-family schemes.

\section{CESE Schemes with Artificial Dissipation}

A nondissipative scheme is not practical for problems where shock wave capturing is of concern. Thus, based on the $a$ scheme, some CESE schemes with artificial dissipation have been proposed, e.g., the $a-\varepsilon$ scheme [4]], the $a-\alpha$ scheme [5], and the Courant-numberinsensitive scheme [6-8]. Generally, these schemes follow the framework of the $a$ scheme but abandon the inverse-time Taylor expansion of Eq. (33) for the flux $F_{C}$. Note that Eq. (31) for the updating of $u_{j}^{n}$ is actually a result of flux balance in the conservation element $(\mathrm{CE})_{j}^{n}$, irrespective of $F_{C}$, and therefore Eq. (31) remains unchanged when extending the nondissipative scheme to a dissipative one.

Necessary dissipation can be introduced into the CESE scheme by updating the spatial derivative $\left(u_{x}\right)_{j}^{n}$ in a different way from Eqs. (32) and (33). In the following, the widely used $a-\alpha$ scheme is outlined as an example. First, $u_{j}^{n}$ is evaluated using Eq. (31). Then, two different estimations for $\left(u_{x}\right)_{j}^{n}$ can be obtained:

$$
\begin{aligned}
&\left(u_{x}^{-}\right)_{j}^{n}= \frac{u_{j}^{n}-\left[u_{j-1 / 2}^{n-1 / 2}+(\Delta t / 2)\left(u_{t}\right)_{j-1 / 2}^{n-1 / 2}\right]}{\Delta x / 2} \\
&\left(u_{x}^{+}\right)_{j}^{n}=\frac{\left[u_{j+1 / 2}^{n-1 / 2}+(\Delta t / 2)\left(u_{t}\right)_{j+1 / 2}^{n-1 / 2}\right]-u_{j}^{n}}{\Delta x / 2}
\end{aligned}
$$

Finally, $\left(u_{x}\right)_{j}^{n}$ is taken as a weighted average of $\left(u_{x}^{-}\right)_{j}^{n}$ and $\left(u_{x}^{+}\right)_{j}^{n}$,

$$
\left(u_{x}\right)_{j}^{n}=W\left(\left(u_{x}^{-}\right)_{j}^{n},\left(u_{x}^{+}\right)_{j}^{n}, \alpha\right)
$$

where $W$ is a weighted-average function with an adjustable parameter $\alpha(\alpha=1$ and $\alpha=2$ are commonly used) expressed as

$$
W\left(x^{-}, x^{+}, \alpha\right)=\frac{\left|x^{+}\right|^{\alpha} x^{-}+\left|x^{-}\right|^{\alpha} x^{+}}{\left|x^{+}\right|^{\alpha}+\left|x^{-}\right|^{\alpha}}
$$

Although the equations in this section apply to 1-D scalar models, important ideas in the early development of the CESE method have been demonstrated. Extensions of the CESE method to 2-D and 3-D cases for Euler, NS, and other physical equations have been well implemented [9-14]. In the extension to NS equations for viscous-flow problems, great efforts have been made to handle the CESE schemes in highly nonuniform and high-aspect-ratio meshes for the purpose of resolving boundary layers.

\section{Recent Progress in CESE Schemes}

Increasing attention to complex problems such as multiphase flows, high-speed aerodynamics, and detonation simulations calls for further improvements to the abilities of existing numerical schemes to capture strong discontinuities, handle complex geometries, and resolve fine flow structures. Accordingly, recent works on CESE schemes have made progress in the following aspects.

\section{A. Upwind CESE Schemes}

The aforementioned CESE schemes are categorized as central schemes, in which the upwind numerical flux technique is not used. Recently, Shen et al. [26], Shen and Wen [27,28], Shen et al. [29], and Shen and Parsani [30] proposed characteristic-based upwind CESE schemes for capturing contact discontinuities (e.g., material interfaces) with improved accuracy and robustness.

The upwind CESE scheme follows the basic ideas of the nondissipative core scheme as much as possible, but some necessary dissipation is introduced by an upwind procedure that is in good accordance with physical processes. For illustrative purposes, the 1-D scalar equation [Eq. (10)] is again considered. The same mesh as shown in Fig. 1 is adopted, and the arrangement of CEs is preserved. However, the definition of the SEs needs to be modified, as shown in Fig. 7. Compared with (SE) ${ }_{j}^{n}$ in Fig. $\underline{4}$, the new $(\mathrm{SE})_{j}^{n}$ becomes a rectangle and no longer contains any part that allows for $t<t_{n}$. With such a modification, it should be noted that Eqs. (17-32) can still be derived by the same procedures as presented in Sec. III.B.

The difference between the upwind CESE scheme and the $a$ scheme lies in the treatment of $F_{C}$ [the average flux through
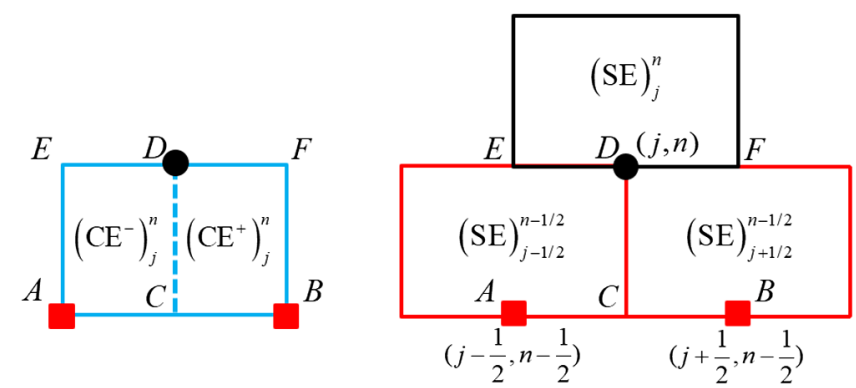

Fig. 7 Definitions of CE and SE for upwind CESE schemes. 
the interface $C D$ between sub-CEs $\left(\mathrm{CE}^{-}\right)_{j}^{n}$ and $\left.\left(\mathrm{CE}^{+}\right)_{j}^{n}\right]$. Instead of using the inverse-time Taylor expansion [Eq. (33)], the upwind CESE scheme approximates $F_{C}$ as

$$
F_{C}=f\left(u_{j}^{n-1 / 4}\right)
$$

where $u_{j}^{n-1 / 4}$ is the value of $u$ at the midpoint of $C D$. In the present upwind CESE scheme, $u_{j}^{n-1 / 4}$ is derived from known data at time $t_{n-1 / 2}$ and can be taken as the solution of the Riemann problem with discontinuous initial data on the left and right sides of $C D$, which can be provided by Taylor expansions in $(\mathrm{SE})_{j-1 / 2}^{n-1 / 2}$ and $(\mathrm{SE})_{j+1 / 2}^{n-1 / 2}$ :

$$
\begin{aligned}
& \left(u_{L}\right)_{j}^{n-1 / 4}=U_{L}+\frac{\Delta x}{4}\left(u_{x}\right)_{j-1 / 2}^{n-1 / 2}+\frac{\Delta t}{4}\left(u_{t}\right)_{j-1 / 2}^{n-1 / 2} \\
& \left(u_{R}\right)_{j}^{n-1 / 4}=U_{R}-\frac{\Delta x}{4}\left(u_{x}\right)_{j+1 / 2}^{n-1 / 2}+\frac{\Delta t}{4}\left(u_{t}\right)_{j+1 / 2}^{n-1 / 2}
\end{aligned}
$$

where $U_{L}$ and $U_{R}$ are given by Eqs. (19) and (21). For the purpose of suppressing oscillations, slope reconstructions with proper limiters are required when using Eq. (39) in cases of strong discontinuities. After $F_{C}$ is obtained, the spatial derivative $\left(u_{x}\right)_{j}^{n}$ can be calculated using Eq. (32). Note that the time-marching scheme in Eq. (31) for $u_{j}^{n}$ is not affected by the aforementioned upwind procedure.

When the upwind CESE scheme is applied to a system of multiple equations (e.g., the Euler equations), the local Riemann problem becomes more complex than that in a scalar case. Therefore, approximate Riemann solvers [e.g., the Harten, Lax, and van Leer [31], contact-restoring Harten, Lax, and van Leer (HLLC) [32], and
Roe [33] Riemann solvers) or other upwind techniques (e.g., local Lax-Friedrichs flux [30]) can be chosen to compute the flux vector at the interfaces between sub-CEs. In multidimensional problems [27], rotated Riemann solvers are preferred for optimizing the direction of the local Riemann problem. So far, the upwind CESE scheme has been extended to multidimensional structured/unstructured meshes for compressible Euler, Navier-Stokes [30], and magnetohydrodynamics (MHD) [34] equations.

\section{B. CESE Schemes on Arbitrary Meshes}

Due to the complexity of geometric configurations and the necessity for local mesh refinements, an optimal computational grid for a 2-D/3-D practical problem may be structured, unstructured, or even hybrid. If conservation elements and solution elements can be defined in a general manner that is suitable for various meshing strategies, the extension of CESE schemes to practical applications will be greatly facilitated [35-37].

Recently, Shen et al. [37] proposed a novel method to construct CEs on arbitrary meshes. Consider a 2-D spatial mesh consisting of mixed quadrilateral and triangular cells. A representative part of this mesh is illustrated by Fig. 8a. The CE for grid node $V_{1}$ at time level $n$ is shown in Fig. 8b. The CEs for cell centers $C_{1}$ and $C_{2}$ at time level $n+1 / 2$ are shown in Figs. $\underline{8 c}$ and $\underline{8 d}$, respectively. Not only the grid nodes and the cell centers but also the midpoints of the cell edges are involved in the definitions of CEs and sub-CEs. Naturally, a CE is split into at least three sub-CEs, and each of these sub-CEs is a quadrangular prism.

Based on the preceding definition of conservation elements for arbitrary meshes, corresponding CESE schemes can be either central or upwind. If the upwind treatment is adopted, the definition of

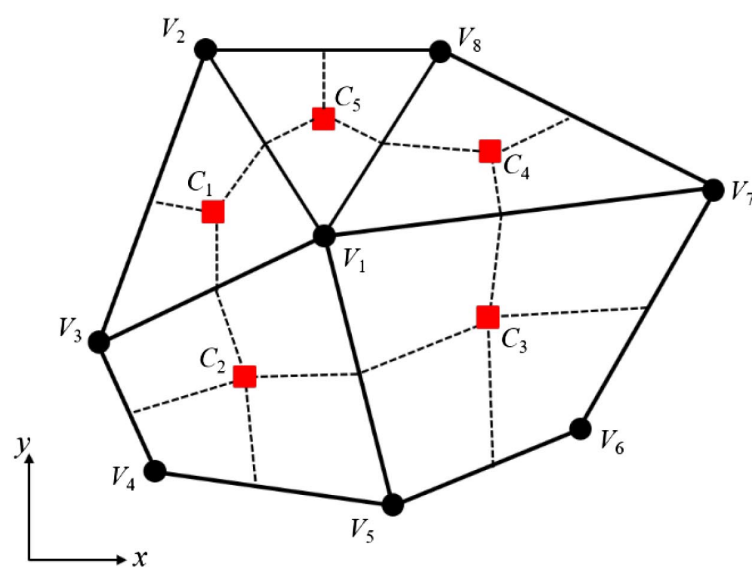

a) A hybrid mesh

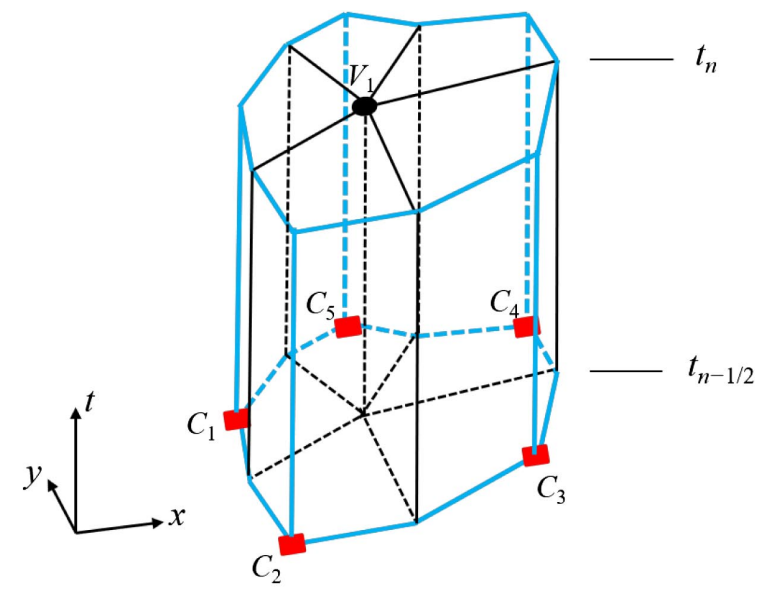

b) $\mathrm{CE}\left(V_{1}\right)$ and its five sub-CEs

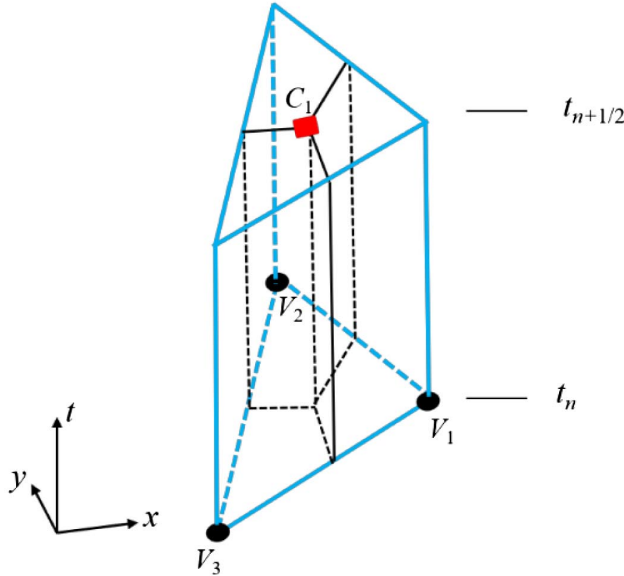

c) $\mathrm{CE}\left(C_{1}\right)$ and its three sub-CEs

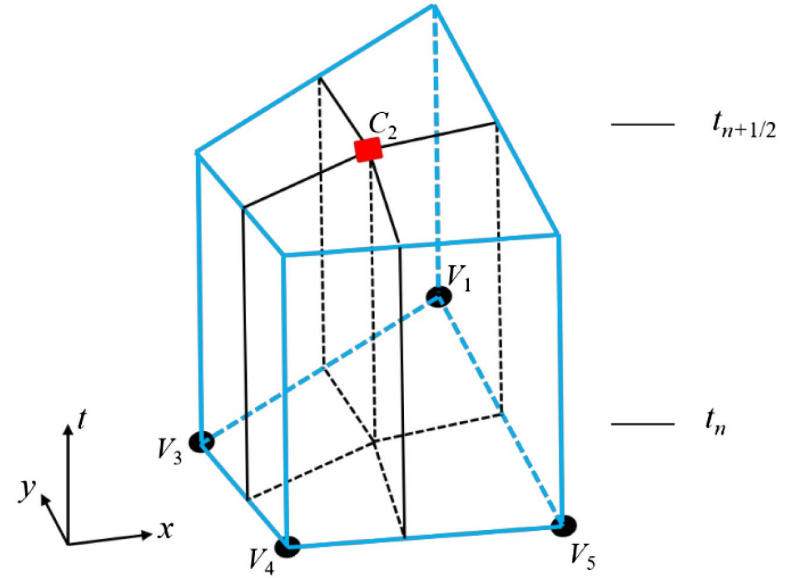

d) $\mathrm{CE}\left(C_{2}\right)$ and its four sub-CEs

Fig. 8 Definition of conservation elements in CESE schemes based on a hybrid mesh. 
solution elements will follow the method shown in Fig. 7. Otherwise, solution elements are defined similarly to those in Fig. 4. See Ref. [37] for a detailed illustration.

Here, the second-order upwind CESE scheme on an unstructured hybrid mesh is presented briefly. Consider the 2-D NS equations [Eq. (1)] and focus on the step from $t_{n-1 / 2}$ to $t_{n}$. In this step, the unknowns for a typical grid node $V_{i}$ include the conserved variables and their spatial derivatives at point $V_{i}$ and time $t_{n}$, which are denoted by $\boldsymbol{U}_{i}^{n},\left(\boldsymbol{U}_{x}\right)_{i}^{n}$, and $\left(\boldsymbol{U}_{y}\right)_{i}^{n}$. The construction of the CE for each grid node $V_{i}$ is shown in Fig. 8b. For the $m$ th sub-CE of $\mathrm{CE}\left(V_{i}\right)$, denoted by $\mathrm{CE}_{m}\left(V_{i}\right)(m=1,2, \ldots, M)$, as shown in Fig. 9, applying Eq. (9) to this sub-CE yields a flux-balancing equation

$$
\begin{aligned}
\boldsymbol{U}_{m}^{*} S_{m} & =\boldsymbol{U}_{m} S_{m}-\frac{\Delta t}{2} \sum_{l=1}^{2}\left(\Delta y_{m}^{(l)} \boldsymbol{F}_{m}^{(l)}-\Delta x_{m}^{(l)} \boldsymbol{G}_{m}^{(l)}\right) \\
& +\frac{\Delta t}{2}\left(L_{m-1} \hat{\boldsymbol{F}}_{m-1}-L_{m} \hat{\boldsymbol{F}}_{m}\right)
\end{aligned}
$$

where $S_{m}$ is the base area of $\mathrm{CE}_{m}\left(V_{i}\right)$; and $\boldsymbol{U}_{m}^{*}, \boldsymbol{U}_{m},\left(\boldsymbol{F}_{m}^{(1)}, \boldsymbol{G}_{m}^{(1)}\right)$, and $\left(\boldsymbol{F}_{m}^{(2)}, \boldsymbol{G}_{m}^{(2)}\right)$ are average values over quadrilaterals $A B V_{i} D, C_{m} G H E$, $A D E C_{m}$, and $A B G C_{m}$, respectively. The definitions of $\boldsymbol{U}, \boldsymbol{F}$, and $\boldsymbol{G}$ are given by Eqs. (2-7); and $\boldsymbol{U}_{m},\left(\boldsymbol{F}_{m}^{(1)}, \boldsymbol{G}_{m}^{(1)}\right)$, and $\left(\boldsymbol{F}_{m}^{(2)}, \boldsymbol{G}_{m}^{(2)}\right)$ can be obtained using the Taylor expansion in $\operatorname{SE}\left(C_{m}\right)$. The parameter $L_{m}$ is the length of $G H$, and $\hat{\boldsymbol{F}}_{m}$ is the flux through the interface $B G H V_{i}$ between $\mathrm{CE}_{m}\left(V_{i}\right)$ and $\mathrm{CE}_{m+1}\left(V_{i}\right)$. The interfacial flux $\hat{\boldsymbol{F}}_{m}$ can be calculated based on the data constructed from $\operatorname{SE}\left(C_{m}\right)$ and $\operatorname{SE}\left(C_{m+1}\right)$, with an upwind technique used for calculating its inviscid part. Hence, $\boldsymbol{U}_{m}^{*}(m=1,2, \ldots, M)$ can be obtained from Eq. (40) for each sub-CE affiliated to $\operatorname{CE}\left(V_{i}\right)$.

With values of $\boldsymbol{U}_{m}^{*}(m=1,2, \ldots, M)$, algebraic equations for unknowns $\boldsymbol{U}_{i}^{n}$, $\left(\boldsymbol{U}_{x}\right)_{i}^{n}$, and $\left(\boldsymbol{U}_{y}\right)_{i}^{n}$ can be established. The top surface of $\operatorname{CE}\left(V_{i}\right)$, which is a polygonal element at time level $n$ (see Fig. 10), consists of $M$ quadrilateral subelements. The $m$ th quadrilateral is the top surface of $\mathrm{CE}_{m}\left(V_{i}\right)$, as shown in Fig. 9. The centroid of the polygonal element is denoted by point $G_{i}$, and the centroid of the $m$ th quadrilateral is point $G_{m}(m=1,2, \ldots, M)$. In this second-order CESE scheme, a piecewise linear assumption is adopted to approximate $\boldsymbol{U}(x, y, t)$ within $\operatorname{SE}\left(V_{i}\right)$. As a result, $\boldsymbol{U}_{m}^{*}(m=1,2, \ldots, M)$ can be interpreted as the value of $\boldsymbol{U}$ at time level $n$ at point $G_{m}$, and $\boldsymbol{U}^{*}\left(G_{i}\right)$ is the average of $\boldsymbol{U}\left(x, y, t_{n}\right)$ over the polygonal element. Based on Eq. (40), the area-weighted average of $\boldsymbol{U}_{m}^{*}(m=$ $1,2, \ldots, M)$ yields the explicit time-marching scheme for $\boldsymbol{U}^{*}\left(G_{i}\right)$ :

$\boldsymbol{U}^{*}\left(G_{i}\right)=\left[\sum_{m=1}^{M} \boldsymbol{U}_{m} S_{m}-\frac{\Delta t}{2} \sum_{m=1}^{M} \sum_{l=1}^{2}\left(\Delta y_{m}^{(l)} \boldsymbol{F}_{m}^{(l)}-\Delta x_{m}^{(l)} \boldsymbol{G}_{m}^{(l)}\right)\right] / \sum_{m=1}^{M} S_{m}$

Note that all fluxes through the interfaces between neighboring sub-CEs are canceled, and Eq. (41) is the direct result of conservation laws over the entire $\operatorname{CE}\left(V_{i}\right)$.

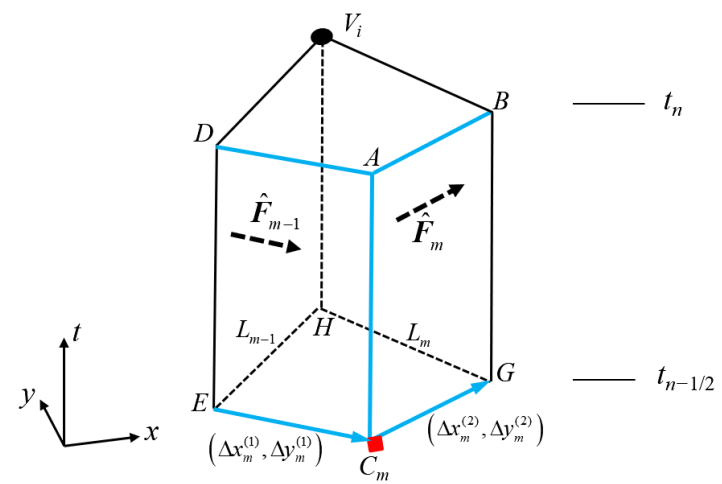

Fig. $9 \mathrm{CE}\left(V_{i}\right)$ : the $m$ th sub-CE belonging to $\mathrm{CE}\left(V_{i}\right), m=1,2, \ldots, M$.

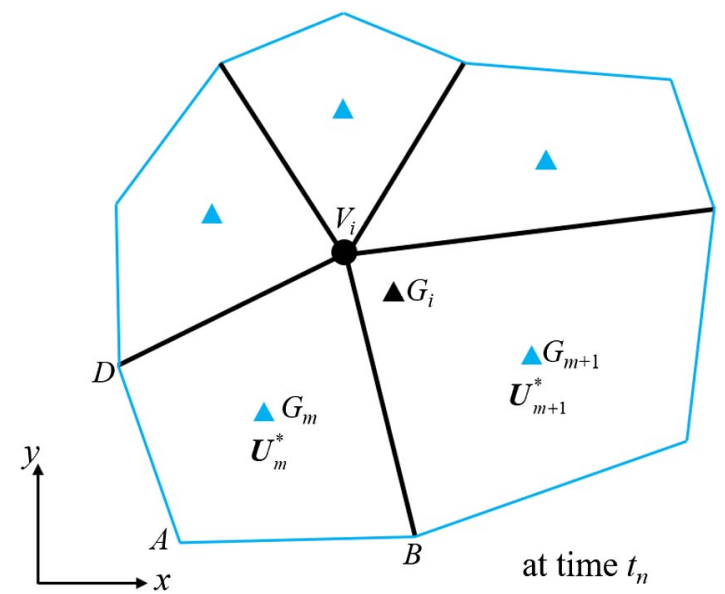

Fig. 10 Top surface of $\operatorname{CE}\left(V_{i}\right)$ : a polygonal element consisting of $M$ quadrilateral subelements.

By using the first-order Taylor expansion inside $\operatorname{SE}\left(V_{i}\right)$, a set of linear equations of $\left(\boldsymbol{U}_{x}\right)_{i}^{n}$ and $\left(\boldsymbol{U}_{y}\right)_{i}^{n}$ can be established as

$$
\begin{gathered}
\left(\boldsymbol{U}_{x}\right)_{i}^{n} \delta x_{m}+\left(\boldsymbol{U}_{y}\right)_{i}^{n} \delta y_{m}=\boldsymbol{U}_{m}^{*}-\boldsymbol{U}^{*}\left(G_{i}\right) \\
\left(\boldsymbol{U}_{x}\right)_{i}^{n} \delta x_{m+1}+\left(\boldsymbol{U}_{y}\right)_{i}^{n} \delta y_{m+1}=\boldsymbol{U}_{m+1}^{*}-\boldsymbol{U}^{*}\left(G_{i}\right)
\end{gathered}
$$

for each $m$, where $\delta x_{m}=x\left(G_{m}\right)-x\left(G_{i}\right)$, and $\delta y_{m}=$ $y\left(G_{m}\right)-y\left(G_{i}\right)$. Therefore, a total of $M$ sets of $\left(\boldsymbol{U}_{x}\right)_{i}^{n}$ and $\left(\boldsymbol{U}_{y}\right)_{i}^{n}$ can be obtained by solving linear equations (42) and (43) for $m=1,2, \ldots, M$. As suggested by Shen et al. [37], a weightedaverage function can be employed to determine an optimal set of derivatives $\left(\boldsymbol{U}_{x}\right)_{i}^{n}$ and $\left(\boldsymbol{U}_{y}\right)_{i}^{n}$, with consideration of the shock-capturing capability as well as the accuracy in smooth regions. Note that the centroid $G_{i}$ does not necessarily coincide with the node $V_{i}$. By using the first-order Taylor expansion

$$
\boldsymbol{U}_{i}^{n}=\boldsymbol{U}^{*}\left(G_{i}\right)+\left(\boldsymbol{U}_{x}\right)_{i}^{n}\left[x\left(V_{i}\right)-x\left(G_{i}\right)\right]+\left(\boldsymbol{U}_{y}\right)_{i}^{n}\left[y\left(V_{i}\right)-y\left(G_{i}\right)\right]
$$

the value of $\boldsymbol{U}_{i}^{n}$ can be updated.

\section{High-Order CESE Schemes}

High-order schemes enable a numerical simulation to accurately resolve fine structures in the physical problem at a relatively low computational cost. Compared with other numerical methods, the CESE method demonstrates two attractive features that are favorable for high-order extensions:

1) The highly compact stencil in the original CESE scheme need not be extended when constructing high-order schemes.

2) The temporal and spatial accuracies can be improved simultaneously [37-41].

Nominally, a CESE scheme based on a first-order Taylor expansion in the solution elements is regarded as a second-order CESE scheme. Recently, Shen et al. [37] proposed arbitrary-order CESE schemes on hybrid meshes, which is an extension of the method of Liu and Wang [38]. Yang et al. [39] then elaborated on this methodology for magnetohydrodynamic simulations. To construct an $n$ th-order CESE scheme $(n>2)$, the space-time fluxes in Eq. (9) need to be approximated by $(n-1)$ th-order Taylor expansions in each SE. Consequently, variables that need to be stored and updated at each solution point include $\boldsymbol{U}$ as well as its spatial derivatives up to the $(n-1)$ th-order, and some unknown high-order derivatives will appear in the integral equation [Eq. (9)] for each CE. Thus, high-order derivatives need to be updated before evaluating $\boldsymbol{U}$ and its first-order derivatives. In the method of Shen et al. [37], these high-order derivatives are updated in a descending sequence from the highest order to the second order. Detailed descriptions of the time-marching scheme for high-order derivatives can be found in Ref. [39]. 
For the purpose of illustration, the third-order CESE scheme of Shen et al. [37] for the 1-D scalar equation [Eq. (10)] is described as follows. With the same definition of computational mesh and CE (see Figs. 1 and 3 ), the integral conservation law in the form of Eq. (9) is applied to the conservation element $(\mathrm{CE})_{j}^{n}$. In this third-order scheme, the second-order Taylor expansion is used to construct $u(x, t)$ and $f(x, t)$ within each SE (see Figs. 4 and 5 ). As a result, the discrete equation for unknowns at mesh node $(j, \bar{n})$ is derived as

$$
u_{j}^{n} \Delta x+\left(u_{x x}\right)_{j}^{n} \frac{\Delta x^{3}}{24}=\mathcal{U}_{L}+\mathcal{U}_{R}+\mathcal{F}_{L}-\mathcal{F}_{R}
$$

where

$$
\begin{gathered}
\mathcal{U}_{L}=\frac{\Delta x}{2} u_{j-1 / 2}^{n-1 / 2}+\frac{\Delta x^{2}}{8}\left(u_{x}\right)_{j-1 / 2}^{n-1 / 2}+\frac{\Delta x^{3}}{48}\left(u_{x x}\right)_{j-1 / 2}^{n-1 / 2} \\
\mathcal{U}_{R}=\frac{\Delta x}{2} u_{j+1 / 2}^{n-1 / 2}-\frac{\Delta x^{2}}{8}\left(u_{x}\right)_{j+1 / 2}^{n-1 / 2}+\frac{\Delta x^{3}}{48}\left(u_{x x}\right)_{j+1 / 2}^{n-1 / 2} \\
\mathcal{F}_{L}=\frac{\Delta t}{2} f_{j-1 / 2}^{n-1 / 2}+\frac{\Delta t^{2}}{8}\left(f_{t}\right)_{j-1 / 2}^{n-1 / 2}+\frac{\Delta t^{3}}{48}\left(f_{t t}\right)_{j-1 / 2}^{n-1 / 2} \\
\mathcal{F}_{R}=\frac{\Delta t}{2} f_{j+1 / 2}^{n-1 / 2}+\frac{\Delta t^{2}}{8}\left(f_{t}\right)_{j+1 / 2}^{n-1 / 2}+\frac{\Delta t^{3}}{48}\left(f_{t t}\right)_{j+1 / 2}^{n-1 / 2}
\end{gathered}
$$

Note that at time level $n-1 / 2, u, u_{x}$, and $u_{x x}$ are already known. Moreover, first- and second-order derivatives with respect to time $t$ (e.g. $f_{t}, f_{t t}, u_{t}, u_{t t}$, and $u_{x t}$ ) at time level $n-1 / 2$ can be calculated by using the Cauchy-Kowalewski procedure, as shown in Eqs. (24-26). Hence, the right-hand-side terms in Eq. (45) can be obtained explicitly.

As suggested in Ref. [37], before updating $u_{j}^{n}$ and $\left(u_{x}\right)_{j}^{n}$, the highest-order spatial derivative $\left(u_{x x}\right)_{j}^{n}$ can be calculated first. A central difference is used to estimate $\left(u_{x x}\right)_{j}^{n}$ as

\section{Some Features of CESE Schemes}

In this section, some remarks are made on the numerical characteristics of the CESE schemes described in Secs. III and IV. Simulations of two simple but canonical problems are performed to demonstrate the capabilities of the CESE method in comparison with established numerical approaches. The stability and efficiency of various CESE schemes are discussed. In particular, a von Neumann analysis for the second-order upwind CESE scheme and an estimation of the data storage in the CESE method are provided.

\section{A. Accuracy}

Generally, the accuracy of a CESE scheme depends on the order of the Taylor expansions that are used to approximate unknowns and fluxes within each solution element (i.e., the degree of the approximation polynomials). If the $(M-1)$ th-order Taylor expansions are used, the theoretical order of accuracy of the scheme is $M$ in space and time. The most frequently used CESE schemes, including the central and upwind types, are second-order schemes.

A feature of the CESE scheme is its ability to capture shock waves and contact discontinuities with high resolution. In this section, two canonical flow problems are numerically simulated by CESE and other schemes to demonstrate the accuracy of CESE schemes. First, Sod's shock-tube problem [42] is solved by a CESE method and a finite volume method (FVM) simultaneously. The CESE code is a 1-D implementation of the second-order $a-\alpha$ scheme described in Sec. III.C. The FVM counterpart incorporates the second-order monotonic upstream-centered scheme for conservation laws (known as MUSCL) reconstruction, the van Leer limiter, and the HLLC Riemann solver. Both the CESE and the FVM computations employ a uniform mesh with 200 cells and a Courant-Friedrichs-Lewy (CFL) number of 0.9 . Numerical results of the density distribution from $x=-1$ to $x=1$ at time $t=0.5$ are shown in Fig. 11 in comparison with the exact Riemann solution. The CESE scheme resolved a narrower contact discontinuity than the FVM scheme.

$$
\left(u_{x x}\right)_{j}^{n}=\frac{\left[\left(u_{x}\right)_{j+1 / 2}^{n-1 / 2}+(\Delta t / 2)\left(u_{x t}\right)_{j+1 / 2}^{n-1 / 2}\right]-\left[\left(u_{x}\right)_{j-1 / 2}^{n-1 / 2}+(\Delta t / 2)\left(u_{x t}\right)_{j-1 / 2}^{n-1 / 2}\right]}{\Delta x}
$$

Then, $u_{j}^{n}$ can be derived from Eq. (45). Finally, $\left(u_{x}\right)_{j}^{n}$ can be determined by a procedure similar to Eqs. (34-37) in Sec. III.C. However, the first-order Taylor expansions in Eqs. (34) and (프) should be replaced with corresponding second-order Taylor expansions. Thus, two different estimations for $\left(u_{x}\right)_{j}^{n}$ can be obtained as

$$
\left(u_{x}^{-}\right)_{j}^{n}=\frac{u_{j}^{n}-\left[u_{j-1 / 2}^{n-1 / 2}+(\Delta t / 2)\left(u_{t}\right)_{j-1 / 2}^{n-1 / 2}+\left(\Delta t^{2} / 8\right)\left(u_{t t}\right)_{j-1 / 2}^{n-1 / 2}\right]}{\Delta x / 2}
$$

$$
\left(u_{x}^{+}\right)_{j}^{n}=\frac{\left[u_{j+1 / 2}^{n-1 / 2}+(\Delta t / 2)\left(u_{t}\right)_{j+1 / 2}^{n-1 / 2}+\left(\Delta t^{2} / 8\right)\left(u_{t t}\right)_{j+1 / 2}^{n-1 / 2}\right]-u_{j}^{n}}{\Delta x / 2}
$$

Substituting Eqs. (51) and (52) into the weighted-average function in Eq. (36) yields the optimal estimation of the first-order spatial derivative $\left(u_{x}\right)_{j}^{n}$.

Additionally, Bilyeu et al. [40] presented a viable approach to developing high-order CESE schemes, with ideas different from Shen et al. [37]. In Ref. [40], Bilyeu et al. successfully constructed a fourth-order CESE Euler solver for 2-D unstructured meshes, which is an extension of Chang's fourth-order CESE method for the 1-D Burgers equation [41].
At the tail of the rarefaction wave, the CESE results match the exact solution better than the FVM results.

The second problem is the Mach 3 inviscid flow through a 2-D tunnel with a forward-facing step inside. See Ref. [43] for initial conditions, boundary conditions, and geometrical parameters. This case is simulated by the $a-\alpha$ and upwind CESE schemes, as well as a flow solver [44] based on the upwind FVM. All of the three schemes ( $a-\alpha$ CESE, upwind CESE, and upwind FVM) have second-order accuracy in space and time, and they employ the same computational mesh (see Fig. 12a) and the same Courant-Friedrichs-Lewy number of 0.95 . Density fields at time $t=4.0$ are shown in Figs. $12 \mathrm{~b}$ and $12 \mathrm{c}$. In comparison with the upwind FVM scheme, both the $\overline{a-\alpha}$ and upwind CESE schemes can accurately capture the structure of shock waves. Moreover, the upwind CESE scheme can resolve the phenomenon of shear-layer instability (see Fig. 12c), which is not observed in the present FVM results.

To assess the accuracy of the high-order CESE schemes described in Sec. IV.C, the Shu-Osher problem and the double Mach-reflection problem were studied intensively in Ref. [37], where the fourth-order CESE scheme provided a good resolution of fine structures in the flows.

\section{B. Stability}

Numerical stability of the CESE method has been investigated ever since this method was proposed. This review paper examines 


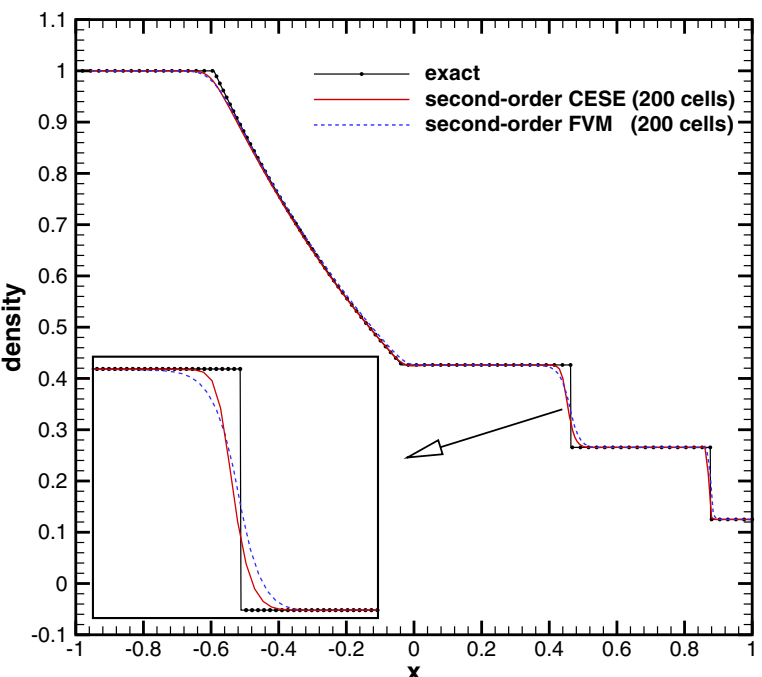

a) CESE result vs FVM result: density

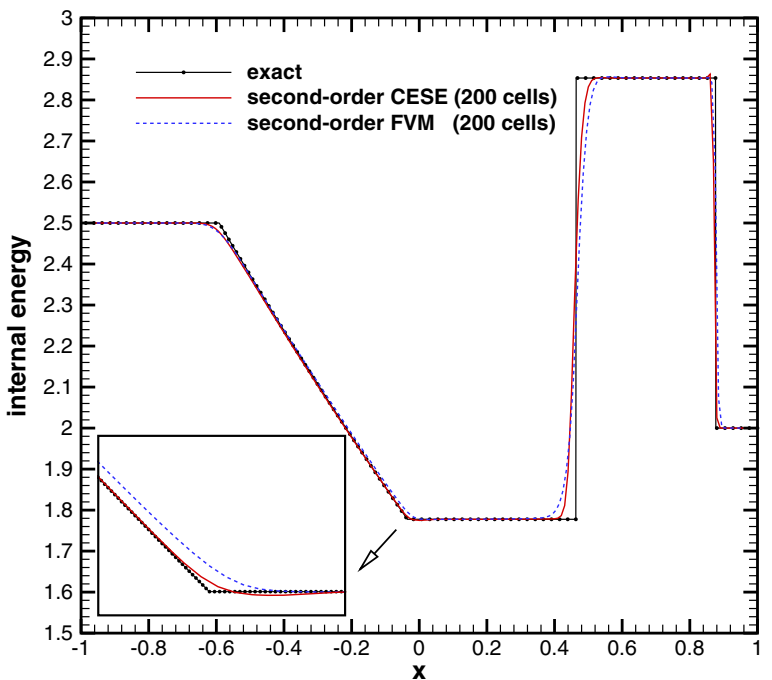

b) CESE result vs FVM result: internal energy

Fig. 11 Numerical results of Sod's shock-tube problem [렬 at dimensionless time $t=0.5$.

several different CESE schemes, and the Courant-Friedrichs-Lewy condition must be satisfied in each of them for the system to remain stable. Consequently, the time-step size used by a CESE scheme has to be restricted according to the upper bound of the stable CFL number for that scheme.

For the second-order $a$ scheme (Sec. III.B) and the second-order $a-\alpha$ scheme (Sec. III.C), the stability condition requires the CFL number to be less than unity. The proof of this conclusion can be found in Refs. [2,18].

For the second-order upwind CESE scheme (Sec. IV.A), the stable upper bound of the CFL number is also equal to one. This stability analysis is not available in the existing literature. Here, a von Neumann analysis on the stability of the second-order upwind CESE scheme is performed. Consider the 1-D linear scalar convection equation, i.e., Eq. (10) with $f=a u$ (here, $a$ is a positive constant). For this equation, the exact solution of the local Riemann problem with initial data [Eq. (39)] can be readily obtained, and thus the interface flux $F_{C}$ [Eq. (38)] is

$$
F_{C}=a\left[U_{L}+\frac{\Delta x}{4}\left(u_{x}\right)_{j-1 / 2}^{n-1 / 2}+\frac{\Delta t}{4}\left(u_{t}\right)_{j-1 / 2}^{n-1 / 2}\right]
$$

With this $F_{C}$, Eqs. (31) and (32) become the explicit expressions of the second-order upwind CESE scheme. Define a solution vector

$$
\boldsymbol{q}_{j}^{n}=\left[\begin{array}{c}
u_{j}^{n} \\
\frac{\Delta x}{4}\left(u_{x}\right)_{j}^{n}
\end{array}\right]
$$

so that Eqs. (31) and (32) can be written in a matrix form

$$
\boldsymbol{q}_{j}^{n}=\boldsymbol{Q}_{L} \boldsymbol{q}_{j-1 / 2}^{n-1 / 2}+\boldsymbol{Q}_{R} \boldsymbol{q}_{j+1 / 2}^{n-1 / 2}
$$

where the coefficient matrices are

$\boldsymbol{Q}_{L}=\frac{1}{2}\left[\begin{array}{cc}1+\nu & 1-\nu^{2} \\ -1+\nu & -1+4 \nu-\nu^{2}\end{array}\right], \quad \boldsymbol{Q}_{R}=\frac{1}{2}\left[\begin{array}{cc}1-\nu & -1+\nu^{2} \\ 1-\nu & -1+\nu^{2}\end{array}\right]$

and $\nu$ is the CFL number, which is defined as

$$
\nu \equiv a \frac{\Delta t}{\Delta x}
$$

Analogously, this scheme can be applied to the half-step marching from time level $n-1$ to time level $n-1 / 2$ :

$$
\boldsymbol{q}_{j-1 / 2}^{n-1 / 2}=\boldsymbol{Q}_{L} \boldsymbol{q}_{j-1}^{n-1}+\boldsymbol{Q}_{R} \boldsymbol{q}_{j}^{n-1}, \boldsymbol{q}_{j+1 / 2}^{n-1 / 2}=\boldsymbol{Q}_{L} \boldsymbol{q}_{j}^{n-1}+\boldsymbol{Q}_{R} \boldsymbol{q}_{j+1}^{n-1}
$$

After substituting Eq. (58) into Eq. (55), a complete time step in the second-order upwind CESE scheme can be formulated as

$$
\boldsymbol{q}_{j}^{n}=\left(\boldsymbol{Q}_{L}\right)^{2} \boldsymbol{q}_{j-1}^{n-1}+\left(\boldsymbol{Q}_{L} \boldsymbol{Q}_{R}+\boldsymbol{Q}_{R} \boldsymbol{Q}_{L}\right) \boldsymbol{q}_{j}^{n-1}+\left(\boldsymbol{Q}_{R}\right)^{2} \boldsymbol{q}_{j+1}^{n-1}
$$

Let $\boldsymbol{q}_{j}^{n}$ be a Fourier component with arbitrary wave number $k$ such that

$$
\boldsymbol{q}_{j}^{n}=\boldsymbol{A}(n, \theta) e^{i j \theta}, \quad(-\pi<\theta \leq \pi)
$$

where $\theta=k \Delta x$ is the reduced wave number, and $i$ is the imaginary unit. Substituting Eq. (60) into Eq. (모) yields

$$
\boldsymbol{A}(n, \theta)=\boldsymbol{M}^{2} \boldsymbol{A}(n-1, \theta)
$$

where

$$
\boldsymbol{M}=\left[\begin{array}{cc}
\cos \frac{\theta}{2}-i \nu \sin \frac{\theta}{2} & i\left(\nu^{2}-1\right) \sin \frac{\theta}{2} \\
i(1-\nu) \sin \frac{\theta}{2} & (2 \nu-1) \cos \frac{\theta}{2}+i\left(\nu^{2}-2 \nu\right) \sin \frac{\theta}{2}
\end{array}\right]
$$

The spectral radius of matrix $\boldsymbol{M}$ is denoted by $\rho(\nu, \theta)$ and is calculated numerically as a function of the CFL number $0 \leq \nu \leq 1$ and the reduced wave number $-\pi<\theta \leq \pi$. The variation of $\rho(\nu, \theta)$ with respect to $\nu$ and $\theta$ is shown in Fig. 13. Provided that the CFL number is less than or equal to unity, it can be seen that $\rho(\nu, \theta) \leq 1$ holds for all wave numbers; thus, this second-order upwind CESE scheme is numerically stable. Additionally, the numerical dissipation in the second-order upwind CESE scheme vanishes as the CFL number approaches zero or one.

For high-order CESE schemes, the stable upper bound of the CFL number depends on the scheme's construction. Chang [41] proposed a novel approach for constructing highly stable high-order CESE schemes using the same space-time stencil as that in the second-order CESE scheme. This construction methodology also retains the CFLnumber constraint for numerical stability (i.e., $\nu \leq 1$ ), which is favorable for explicit time-stepping computations. 


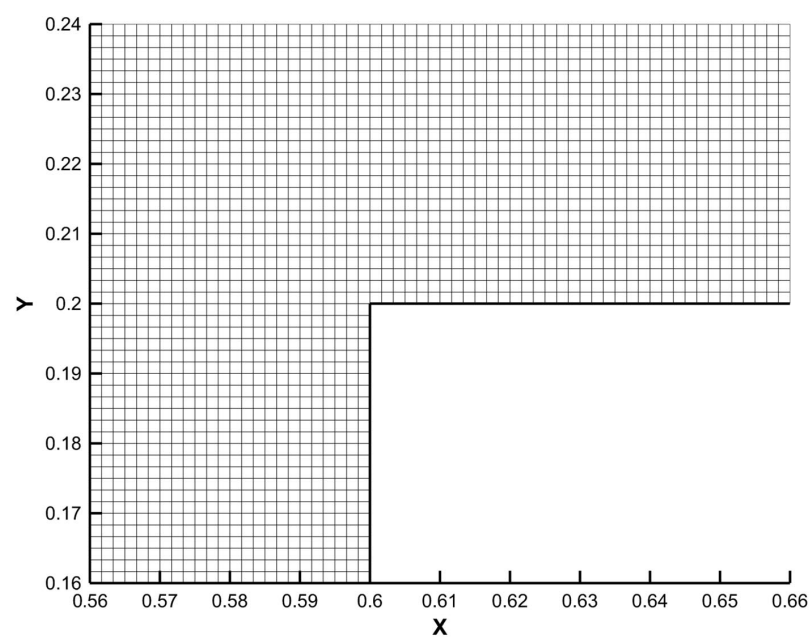

a) The uniform and orthogonal mesh $(1800 \times 600$ cells $)$ used by the different schemes

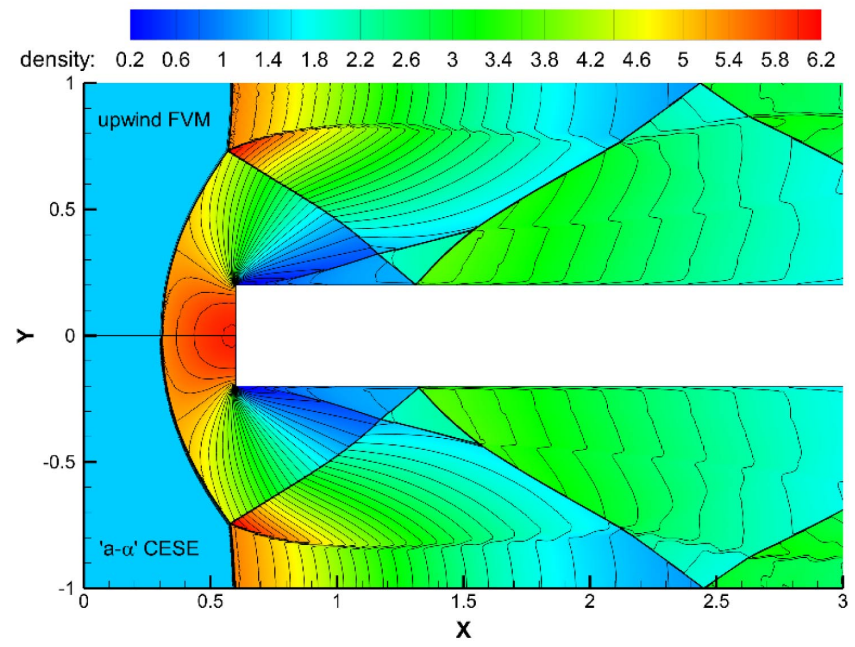

b) a- $\alpha$ CESE result vs FVM result

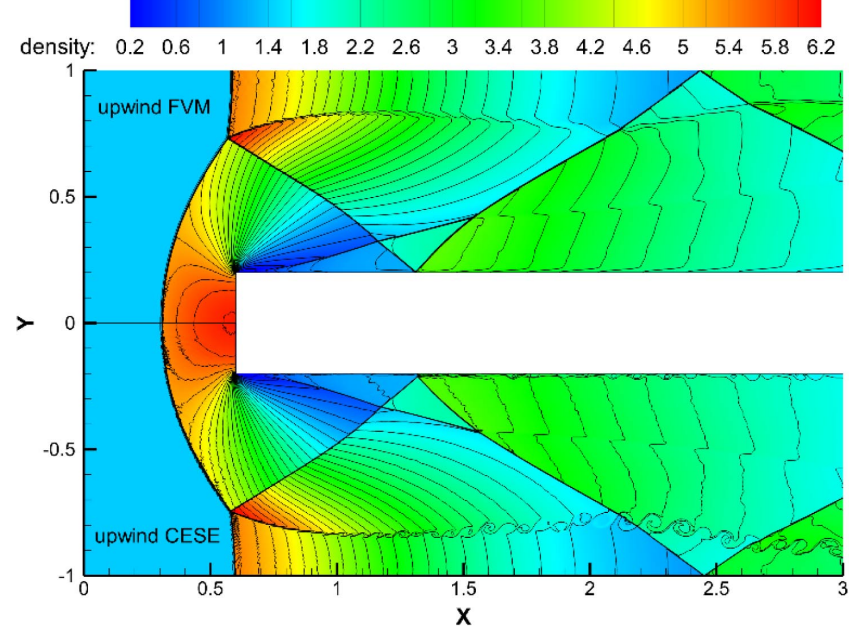

c) Upwind CESE result vs FVM result

Fig. 12 FVM and CESE results for Mach 3 step problem: density contours at time $t=4.0$.

\section{Efficiency}

The CESE method has a highly compact stencil in space and time, as shown in Fig. 2, regardless of the order of the scheme. The compactness of the CESE method is favorable for parallelization.

In a CESE scheme, the number of independent variables that must be stored and updated at each solution point (denoted by $K$ ) is related to the order of accuracy. For a spatially $D$-dimensional $(D=1,2$, or 3 ) and $M$ th-order accurate $(M \geq 2)$ CESE scheme, a precise counting indicates that

$$
K(D, M)= \begin{cases}M, & D=1 \\ M(M+1) / 2, & D=2 \\ M(M+1)(M+2) / 6, & D=3\end{cases}
$$

Note that this number is the same as the number of degrees of freedom for each cell in the discontinuous Galerkin (DG) scheme. Therefore, the memory requirement of a CESE scheme is comparable to that of a DG scheme if the CESE and DG schemes are of the same order and they are used to solve the same problem on the same mesh.

The CESE method requires neither a reconstruction procedure based on a wide stencil nor a multistage time-integration technique. Moreover, central CESE schemes (just like central finite volume and central finite difference schemes) do not include any upwind operations related to flux calculation. Nevertheless, the CESE method uses the Cauchy-Kowalewski procedure to express the temporal derivatives in terms of spatial derivatives. It also takes time to update the spatial derivatives using the algorithms described in Secs. III and IV. The computational speed of the CESE method is determined by the combined effect of all these factors. For example, consider the secondorder $a-\alpha$ CESE and FVM schemes employed in the 1-D shock-tube problem in Sec. V.A. For each scheme, the CPU time consumed by the computational program per time step was measured. The CPU times listed in Table 1 demonstrate the favorable efficiency of the $a-\alpha \mathrm{CESE}$ scheme.

\section{Applications}

Since its inception in the 1990s, the CESE method has been applied to a wide range of scientific and engineering problems. Although CFD problems are the primary objectives of the CESE method, this simple and general method actually fits various PDE systems with physical backgrounds beyond fluid dynamics. This section focuses on the recent highlights of CESE applications in several representative research areas.

\section{A. High-Speed Flows in Aerospace Applications}

The CESE method has been successfully applied to typical supersonic aerodynamic problems, including the supersonic flow over a blunted flat plate [45], Mach reflection of shock waves [46], unsteady viscous flows in rocket nozzles [47], counterflow jets for the reduction of aerothermal load on spacecrafts $[48,49]$, and the flow over roughness elements in a supersonic boundary layer [50].

In recent years, increasing interest in reentry flights and Mars missions has resulted in increasing attention to the field of hypersonic aerothermodynamics. Typically, the flows encountered by hypersonic vehicles are characterized by strong shock waves and thermochemical nonequilibrium effects. To thoroughly understand the complicated flow physics involved and reliably predict aerodynamic heating, numerical studies are essential.

Recently, Wen et al. [51], Shen et al. [52], and Massimi et al. [53] conducted a systematic study of hypersonic chemically reacting nonequilibrium flows over spheres using a 2-D axisymmetric CESE solver based on hybrid meshes. Simulations were performed for three different working gases: nitrogen, air, and carbon dioxide. The vibrational energy relaxation and the dissociation and recombination reactions were taken into account. In particular, the physically consistent coupled vibration-chemistry-vibration (CVCV) [54] model was employed. Here, three flow cases are selected to show the capabilities of the CESE method. The working gases, sphere radii, and freestream conditions of these cases are listed in Table 2. The numerical results of the dimensionless shock standoff distances (the ratio of the standoff distance to the sphere radius) are listed in Table 3 , alongside the experimental and theoretical results of Wen and Hornung [55]. It can be seen that the CESE results agree well with the experimental and theoretical results. Furthermore, the shapes of the shock waves are shown in Fig. 14, where the numerical density contours are 


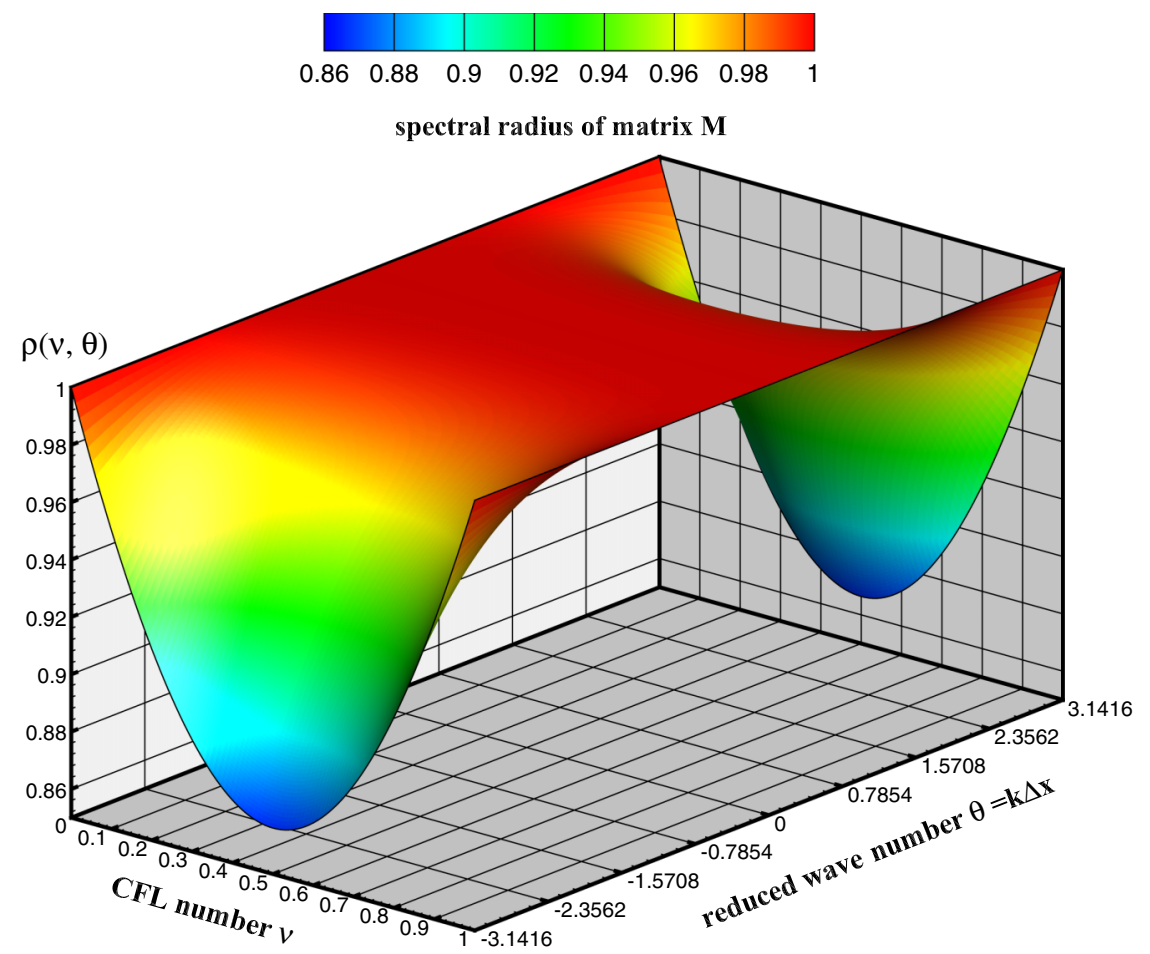

Fig. 13 Spectral radius $\rho(\nu, \theta)$ of matrix $M$ in the von Neumann analysis of the upwind CESE scheme.

overlaid on the experimental finite fringe differential interferograms. The shapes of the numerical bow shocks match the experimental results well.

Another important nonequilibrium process in an atmosphericentry flow is ionization in a high-temperature shock layer. Massimi et al. [56] carried out a numerical investigation of hypersonic ionized flows over rounded nose geometries. A 2-D axisymmetric CESE solver based on hybrid meshes was used to solve the NS equations with the two-temperature seven-species Park model for ionization reactions. The weakly ionized air flow in the Radio Attenuation Measurement (RAM-C II) flight test at a $71 \mathrm{~km}$ altitude [57] was simulated. As shown in Fig. 15, the CESE results of the maximum electron number densities along the direction normal to the vehicle's

Table 1 CPU time per step in the 1-D computation of the shock-tube problem

\begin{tabular}{lc}
\hline \hline Method & CPU time per step, ms \\
\hline Second-order CESE (200 cells) & 0.0125 \\
Second-order FVM (200 cells) & 0.0188 \\
\hline \hline
\end{tabular}

Table 2 Freestream conditions of flow cases selected from Wen and Hornung [55]

\begin{tabular}{lccccc}
\hline \hline Case no. & Gas & Radius, in. & $\rho_{\infty}, \mathrm{kg} / \mathrm{m}^{3}$ & $u_{\infty}, \mathrm{m} / \mathrm{s}$ & $T_{\infty}, \mathrm{K}$ \\
\hline 1 & $\mathrm{~N}_{2}$ & 1.5 & 0.0195 & 5140 & 2200 \\
2 & $\mathrm{Air}$ & 1.0 & 0.0278 & 3930 & 1370 \\
3 & $\mathrm{CO}_{2}$ & 2.0 & 0.0326 & 3490 & 2010 \\
\hline \hline
\end{tabular}

Table 3 Experimental, theoretical, and numerical dimensionless shock standoff distances

\begin{tabular}{lccc}
\hline \hline Case no. & Experiment [55] & Theory [55] & Simulation [51] \\
\hline 1 & 0.100 & 0.095 & 0.100 \\
2 & 0.105 & 0.093 & 0.095 \\
3 & 0.088 & 0.084 & 0.087 \\
\hline \hline
\end{tabular}

surface are in good agreement with the data measured during the flight test [57].

\section{B. Compressible Multifluid Flows}

Multifluid flows involving shock-induced instability and shockaccelerated inhomogeneities play essential roles in a wide variety of problems including, but not limited to, supersonic combustion, inertial confinement fusion, and supernova explosion. Numerical simulations of these complex flows prove to be challenging in the presence of moving and deformable material interfaces, especially for fluids with large differences in their densities or thermodynamic properties. Therefore, a discontinuity-capturing, mass-conserving, and positivity-preserving scheme is desirable for compressible multifluid simulations.

Qamar et al. [58] implemented the CESE method of Chang [2] and Zhang et al. [12] for solving the one- and two-dimensional compressible two-fluid models of Kreeft and Koren [59]. Numerical simulations were performed for gas-liquid Riemann problems and interactions of air shock waves with bubbles containing lighter and heavier gases. In comparison with the nonoscillatory central scheme [60] and the kinetic flux-vector splitting scheme [61], the CESE scheme gives better resolution of discontinuities. Fu et al. [62] applied the CESE method to a two-fluid Richtmyer-Meshkov instability (RMI) investigation. Their numerical results are consistent with the corresponding experimental results [63]. Furthermore, the detailed characteristics (including small vortices along discontinuities, rollup structures, and the evolution of the amplitude of the mixing layer in RMI) are clearly revealed by the CESE simulation. Recently, the upwind CESE method was used extensively in studies of interfacial instabilities [64-67].

In the recent works of Shen et al. [29], Guan et al. [68], and Fan et al. [69], compressible two-fluid flows are described by a volumefraction-based five-equation model [70] coupled with the stiffened gas equation of state [71]. Extensive numerical simulations were carried out using the maximum-principle-satisfying upwind CESE scheme [29], which is an improved version of the upwind CESE scheme presented in Sec. IV. The maximum-principle-satisfying property is achieved by adopting a very simple limiter proposed by Zhang and Shu [72]. Furthermore, the ability to capture contact discontinuities (material interfaces) can be enhanced by employing 


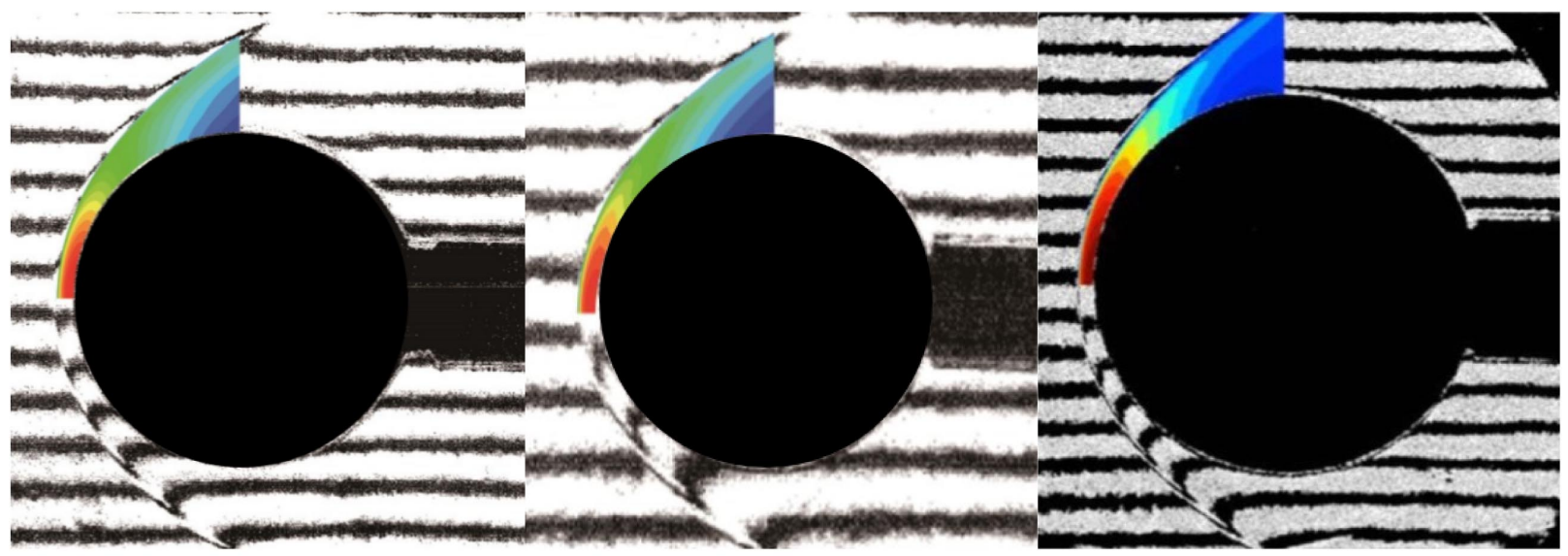

a) Case 1

b) Case 2

c) Case 3

Fig. 14 Comparison of the numerical and experimental shapes of the bow shocks [51]. Reprinted with permission from Elsevier.

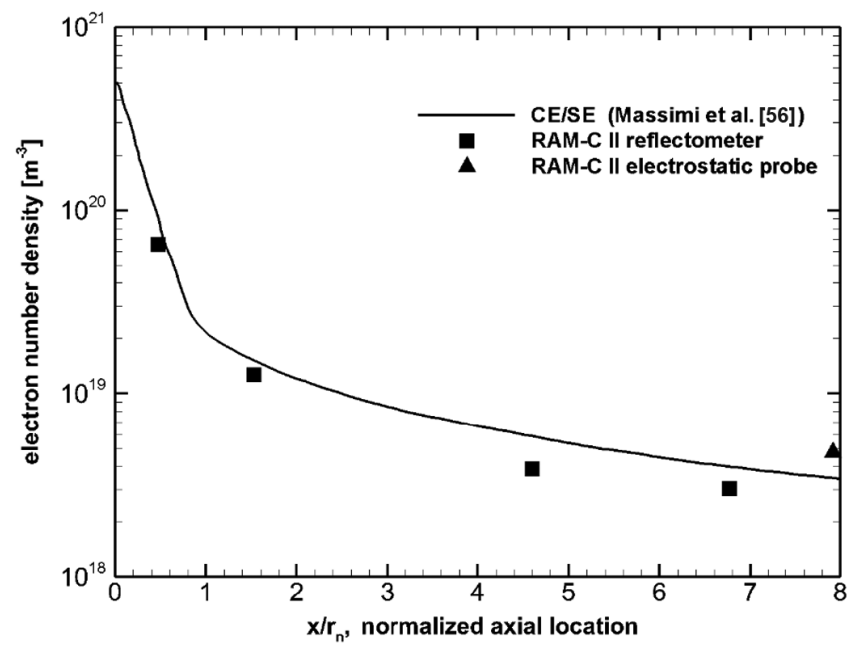

Fig. 15 Comparison of electron number density along the body for RAM-C II flight test at $71 \mathrm{~km}$ altitude.

the HLLC Riemann solver in the upwind procedure. As a result, challenging numerical simulations of the gas-liquid Riemann problem and the RMI on a circular interface were successfully performed, in which the mass of each fluid component is conserved and the positivity of volume fractions is preserved [29].

Here, we review the numerical studies of shock-accelerated inhomogeneous flows conducted by Shen et al. [29], Guan et al. [68], and Fan et al. [69]. Figure 16 shows a schematic of the computation of a shock-bubble interaction [29], in which a planar shock propagates from left to right at Mach 1.22 and impacts a helium bubble with a radius of $25 \mathrm{~mm}$. The morphology of the bubble during its deformation process is shown in Fig. 17. It can be seen that the interface between the different fluids is sharply captured, and vorticities are generated near the interface due to the baroclinicity [73]. To quantitatively validate the CESE method, the trajectories of the upstream point, the jet point, and the downstream point of the bubble are plotted in Fig. 18. A good agreement is observed in the comparison between the CESE results and those obtained by the level-set method [74].

Furthermore, a simulation of a shock-water-column interaction was performed in Ref. [29] to demonstrate the ability of the upwind CESE method to handle gas-liquid interfaces. The setup of this problem resembles that of the shock-bubble interaction (see Fig. 16). However, the simulation of a shock-water-column interaction becomes more challenging due to the high-density ratio and large difference between the thermodynamic properties of air and water. In this case, the radius of the water column was $2.4 \mathrm{~mm}$ and the Mach number of the shock wave was 1.47 . Because of the high density (and thus large inertia) of the water column, the interaction process can actually be separated into two stages: the propagation of pressure waves and the deformation of the water column. At the early stage, as shown in Fig. 19, the shock propagates through the water column as though passing over a rigid cylinder, but the pressure inside the water column changes very quickly and becomes highly heterogeneous. After the incident shock has passed, as shown in Fig. 20, the water is gradually stripped away by the high-speed airflow. Note that the instabilities were captured in detail in this CESE simulation. For quantitative validation, the drag coefficients of the water column at different times were derived from the CESE results. As shown in Fig. 21, there is good agreement with the numerical simulations of Chen [75] and the experimental data of Igra and Takayama [76].

Guan et al. [68] simulated the shock-induced breakup process of a liquid droplet immersed in air. The initial setup of this numerical case is shown in Fig. 16. By following the methodology in Ref. [29], the flowfield inside the liquid droplet was clearly visualized in the CESE results, which indicate that the flow pattern inside the droplet is established soon after the impact by the incident shock and holds steady for a long time after it passes. For the first time, a saddle point was observed in this internal flow pattern. Cases with different incident shock strengths were tested (Fig. 22), and a simple theory was proposed to correlate the stationary position of the saddle point with the Mach number of the incident shock.

A comprehensive study of the jet-formation phenomenon in the interaction of a planar shock with a heavy gas inhomogeneity was

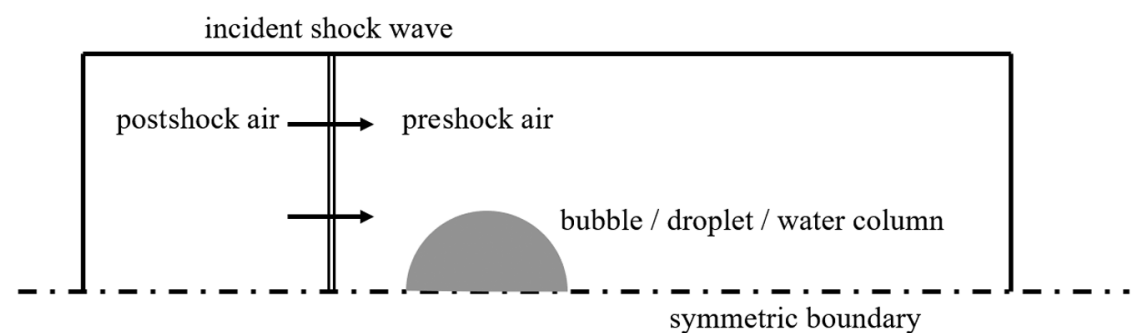

Fig. 16 Schematic of the initial setup for the interaction of an incident shock wave and a gas bubble/water droplet/water column. 


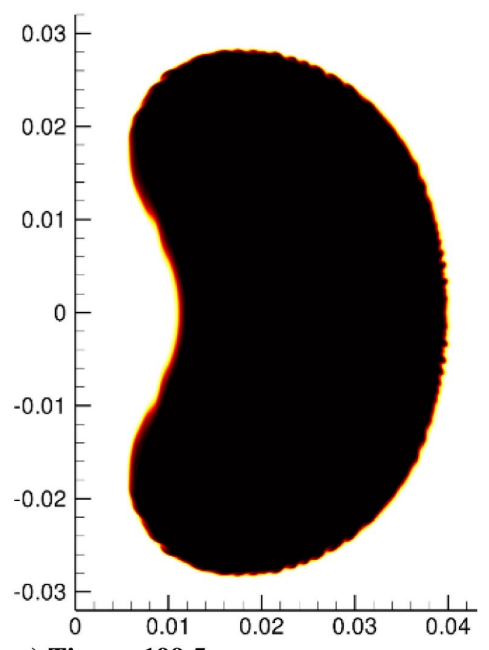

a) Time $=199.5 \mu \mathrm{s}$

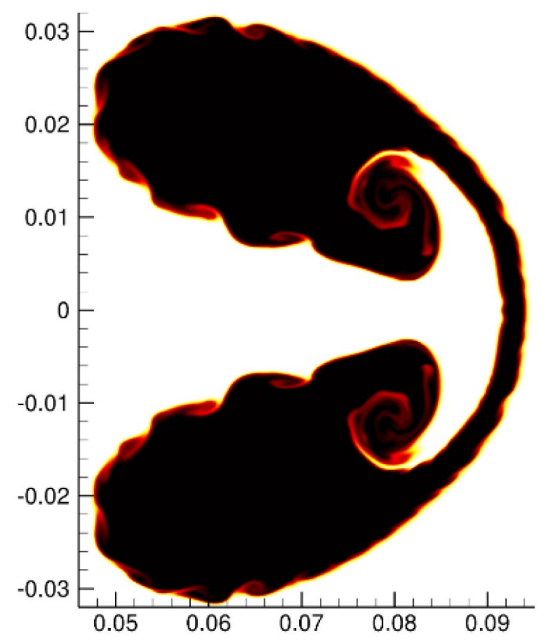

c) Time $=600.6 \mu \mathrm{s}$

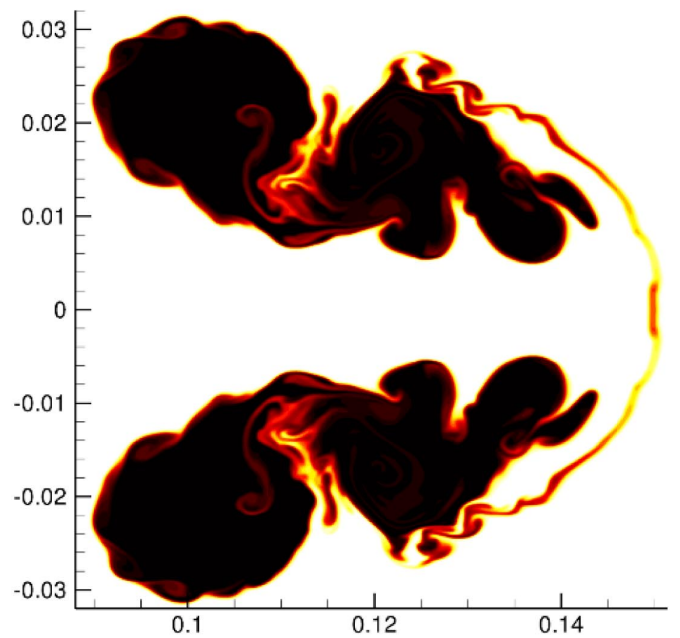

e) Time $=999.5 \mu \mathrm{s}$

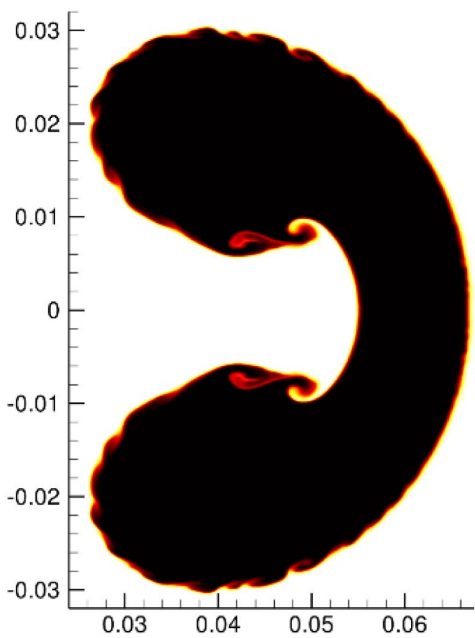

b) Time $=399.7 \mu \mathrm{s}$

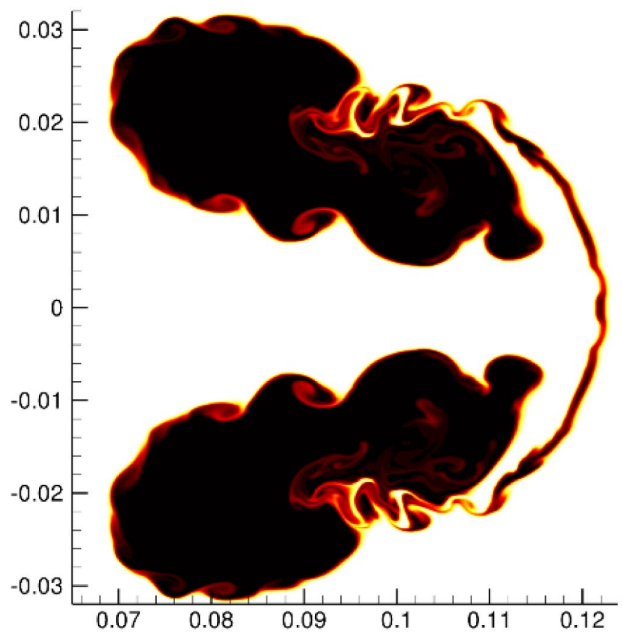

d) Time $=800.2 \mu \mathrm{s}$

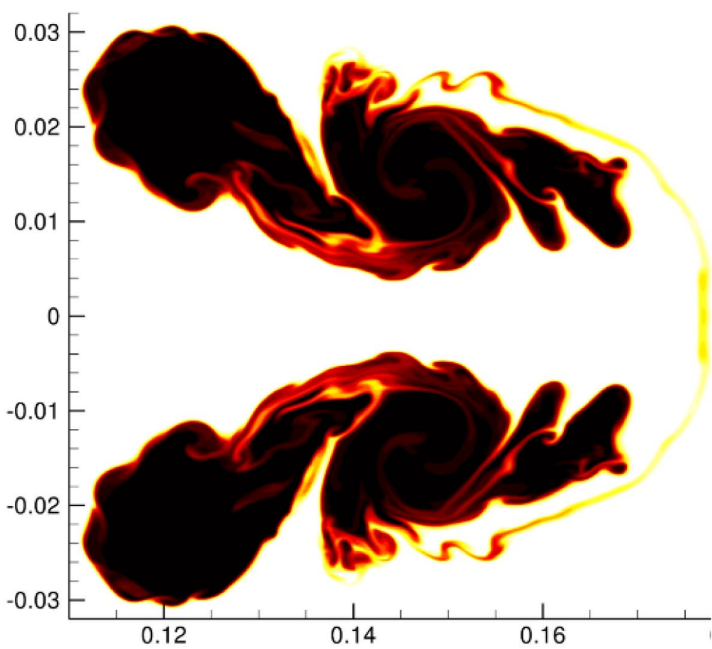

f) Time $=1200.0 \mu \mathrm{s}$

Fig. 17 Deformation history of a helium bubble impacted by a Mach 1.22 shock [29]. Reprinted with permission from Elsevier.

conducted by Fan et al. [69], in which the maximum-principlesatisfying upwind CESE scheme was adopted for numerical simulations. A schematic of the computation setup is shown in Fig. 23. As a validation of this CESE scheme, the interaction between a Mach 1.17 air shock wave and an sulfur hexafluoride $\left(\mathrm{SF}_{6}\right)$ square cylinder (with a length of $56.6 \mathrm{~mm}$ ) was simulated with the aim of reproducing the corresponding experimental images [77]. The numerical results shown in Fig. 24 are in excellent agreement with the experimental images, especially for the morphology of the gas interface, the pattern of the shock system, and the consequent jet formation on the middle leeward surface of the inhomogeneity. Based on the detailed information provided by the CESE simulation, the mechanism of jet formation was revealed, and a geometrical criterion was proposed to determine whether a jet will be formed [69].

Extensive numerical examples in this subsection indicate that the CESE method captures shocks and contact discontinuities sharply without spurious oscillations and proves to be a robust and accurate numerical tool for studies of compressible multifluid flows. 


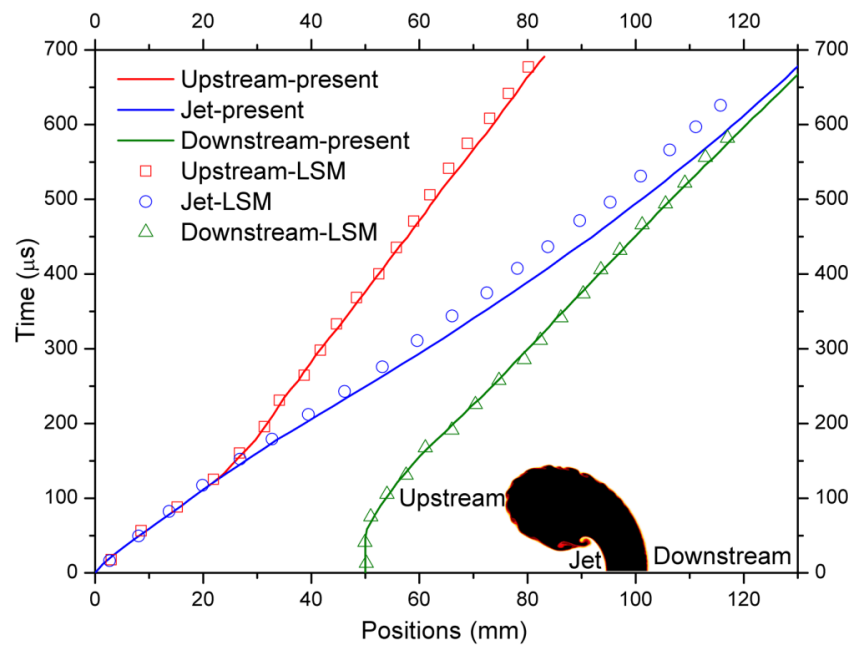

Fig. 18 Position history of the upstream point, the jet point, and the downstream point of the helium bubble [29]. Reprinted with permission from Elsevier.

\section{Detonation Simulation}

A detonation wave is essentially a strong shock wave supported by the chemical energy release behind it [78]. This has been attracting worldwide research interest because of its potential application to propulsion systems. For detonation, in addition to theoretical modeling and experimental observation, an alternative approach is to perform highly resolved and accurate numerical simulations to gain insights into the complex physical processes involved.

An unstable multidimensional structure is inherent in the detonation phenomenon. By using the CESE method to solve reactive Euler equations, Park et al. [79] and Zhang et al. [80] conducted 2-D and 3-D simulations of unstable detonations and crisply resolved the flow features, including the transverse wave structure, triple points, Mach stems, counter-rotating vortices, and unburned pockets. Weng and Gore [81] studied the 3-D flow dynamics in a pulse-detonation engine using the CESE method. Shen et al. [82] applied the CESE method to 3-D Euler equations coupled with a two-step reaction model and investigated the mechanism of 3-D detonation propagation in rectangular ducts.

Recently, Shi et al. [83] noticed that in numerical studies of cellular structures in detonation waves, it was difficult to match the numerical cell size with that observed in experiments. For a hydrogen-oxygen detonation simulation, this discrepancy can be partly attributed to neglect of the vibrational relaxation process when modeling chemical reactions. To illustrate the importance of vibrational nonequilibrium effects on the detonation cell size, the CESE method was used to solve reactive Euler equations under four different scenarios:

1) The whole system is in thermodynamic equilibrium.

2) The vibrational relaxation is considered and the translationalrotational temperature is used as the dominant temperature of the chemical reactions.

3) The vibrational relaxation is considered, whereas Park's twotemperature model [84] is used to account for the effect of vibrational temperature on chemical-reaction rates.

4) A more physically consistent vibration-chemistry-vibration coupling (CVCV) model [54] is adopted. In Fig. 25, cellular detonation patterns corresponding to physical models $1-4$ are visualized with the numerical soot-foil technique [78]. When the Park's twotemperature model [84] or the CVCV model is used, the detonation cell is effectively enlarged, and a more reasonable agreement can be achieved between the numerical cell width and the experimental measurement. Therefore, the vibrational relaxation process in the chemical-reaction models is an important mechanism in gaseous detonation.

Initiation of detonations has been numerically studied using the CESE method. Wang et al. [85] considered implosion as a useful approach to facilitate the detonation initiation process. To analyze the complex structures of converging shock waves and the subsequent detonation initiation, 1-D NS equations with a 24-step chemical model for hydrogen-oxygen detonation and 2-D Euler equations with a one-step chemical model for propane $\left(\mathrm{C}_{3} \mathrm{H}_{8}\right)$-air detonation were solved using the CESE method. The results clearly demonstrate that the CESE method can capture the salient features of complex implosion flows. As a result of shock interactions in the implosion system, a robust detonation wave can be quickly developed.

Recently, Shen and Parsani [86] used the CESE method to investigate the direct initiation of detonation driven by a blast wave. To study the role of multidimensional instabilities in the direct initiation of detonations, CESE simulations were performed for 1-D and 2-D reactive Euler equations. Comparisons between the 1-D and 2-D results indicate that multidimensional effects must be included in a predictive model of the direct initiation of unstable detonations. Heat release and unsteadiness are undoubtedly two competing factors in the failure or success of the initiation of real detonations, but they are accompanied by a third key factor: inherent multidimensional instabilities. As shown in Fig. 26, multidimensional instabilities lead to the formation of strong transverse waves, which on one hand increase the risk of failure of the detonation, and on the other hand give rise to the initiation of local overdriven detonations that enhance the overall self-sustainability of the global process. The competition between these two possible mechanisms plays an important role in the direct initiation of detonations. In Ref. [87], the reinitiation mechanism of a diffracted detonation wave was investigated numerically using the CESE method.

Since liquid or solid fuels are in favor for many propellant applications, understanding the characteristics of two-phase detonations has become important. The CESE method has been successfully extended to solve the gas-liquid and gas-solid detonation problems, and it has proved to be highly accurate and robust. Wang et al. [88] applied the CESE method to gas-droplet detonations under an Eulerian-Eulerian (two-fluid) framework, in which the droplet phase is considered as a continuum medium. Additionally, Wang et al. [89] simulated the explosive synthesis of titania $\left(\mathrm{TiO}_{2}\right)$ nanoparticles using the Eulerian-Eulerian description and a similar CESE scheme as in Ref. [88]. Dong et al. [90] applied the CESE method to a pistondriven detonation in energetic solid granules. In the work of Shen et al. [91], an Eulerian-Lagrangian two-phase flow model was constructed for liquid-fueled detonations, and the CESE method was adopted for the numerical simulations. In the mixtures with lean fuel, the propagation velocity of the two-phase detonation was found to be smaller than that of the equivalent gaseous detonation. This deficit in the detonation velocity is not only due to heat and drag losses in the reaction zone but also the incomplete fuel reaction between the shock wave and the Chapman-Jouguet plane.

Zhang et al. [92] studied the detonation processes in reactive gas-solid-particle mixtures using the CESE method. The two-phase detonation was described under an Eulerian-Lagrangian framework. The motion of the gas can be described by the unsteady, multispecies, reactive Euler equations with interphase interaction terms, whereas the motion of every particle can be tracked by Newton's laws of motion. The surface-kinetic-oxidation and diffusion hybrid combustion models [93] were employed to model the combustion rate of the solid particles. Momentum exchange, heat transfer, and mass exchange between the gas and the particles were considered. With efficient message passing interface parallelization techniques, a CESE simulation code for solving these physical models has been developed. Numerical simulations of 1-D and 2-D aluminum (Al)-air detonations (see Fig. 27) were performed, and comparisons between the monodisperse and polydisperse gas-particle detonations with lognormal particle size distributions were conducted. From the numerical results, it can be seen that most features of monodisperse Al-air detonations, including the double peaks in the gas pressure profile and the kink in the gas temperature profile, disappear in polydisperse detonations. Moreover, in polydisperse detonations, the reaction zones are larger than those in monodisperse cases, which consequently yield larger detonation cell sizes. Since the cellular structures reflect the multidimensional instabilities of detonation propagation, it 


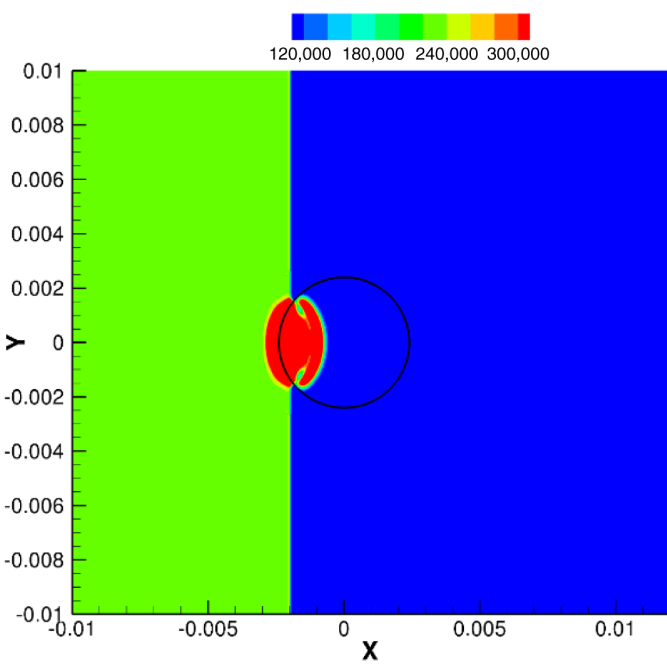

a) Time $=1.85 \mu \mathrm{s}$

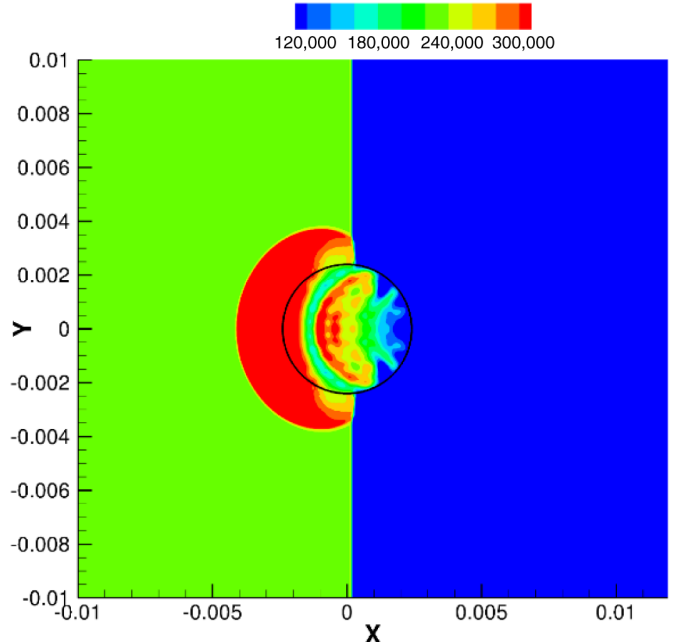

c) Time $=6.15 \mu \mathrm{s}$

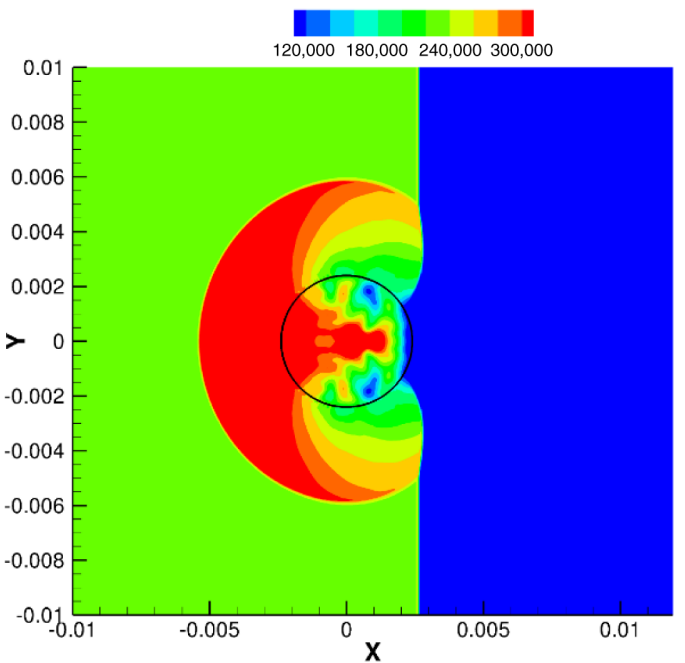

e) Time $=11.1 \mu \mathrm{s}$

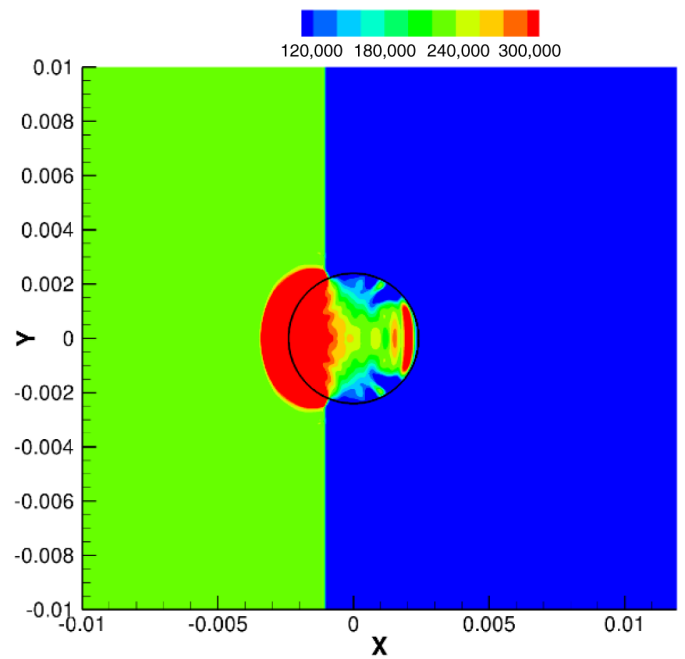

b) Time $=3.69 \mu \mathrm{s}$

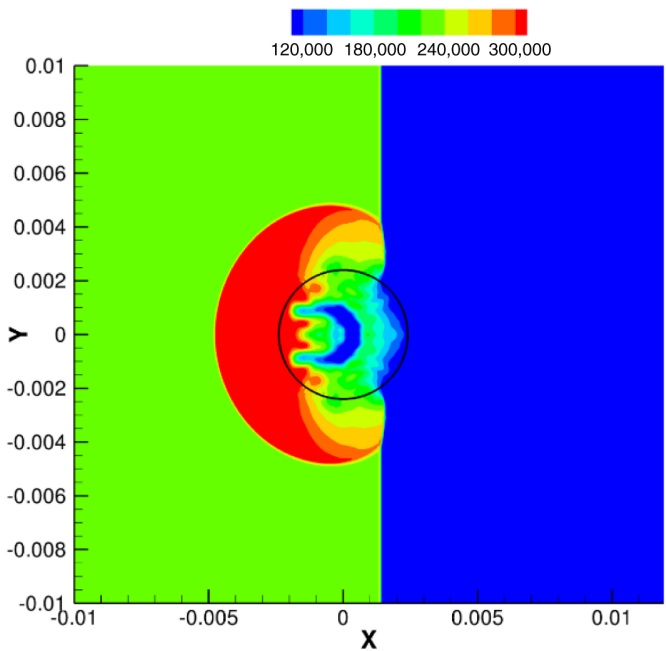

d) Time $=8.61 \mu \mathrm{s}$

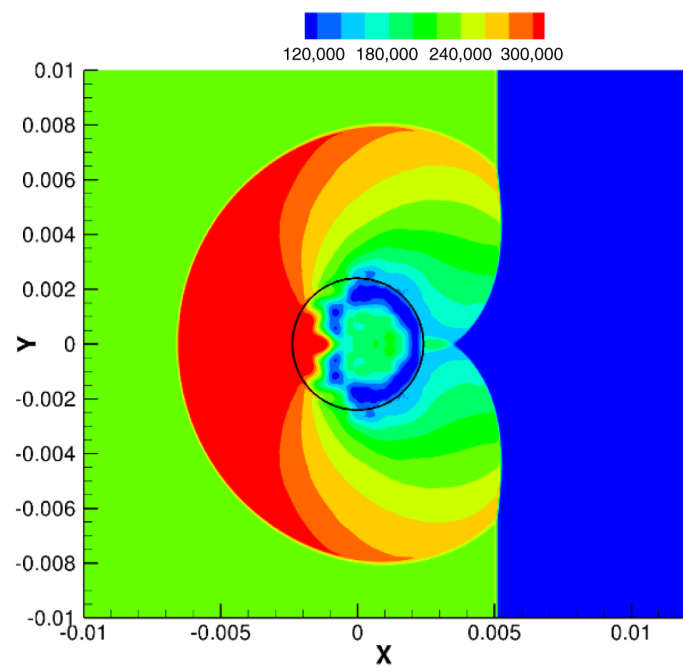

f) Time $=16.0 \mu \mathrm{s}$

Fig. 19 Sequences of pressure contours (in units of pascals) in the early stages of a shock-water-column interaction [29]. Reprinted with permission from Elsevier.

is clearly demonstrated that the particle size distributions affect the multidimensional instabilities of gas-particle detonations.

\section{Computational Aeroacoustics}

By taking advantage of rapid advances in the CFD field, modern aeroacoustic problems can be promisingly solved through highresolution numerical simulations of unsteady compressible Euler or
NS equations. Nevertheless, in a number of computational aeroacoustic problems, the coexistence of both shock waves and small disturbances (acoustic waves) places stringent requirements on the numerical method.

Wang et al. [94] used the CESE method to study the soundshock interaction problem and investigated the accuracy of the CESE method for aeroacoustic problems involving shock waves. 


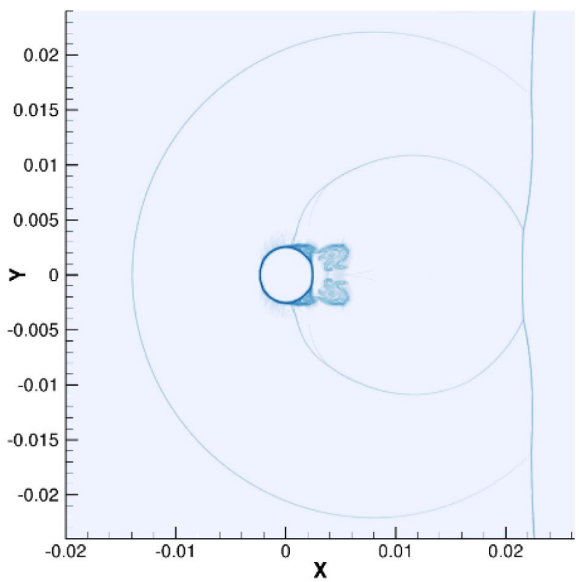

a) Time $=\mathbf{5 0 . 4} \mu \mathrm{s}$

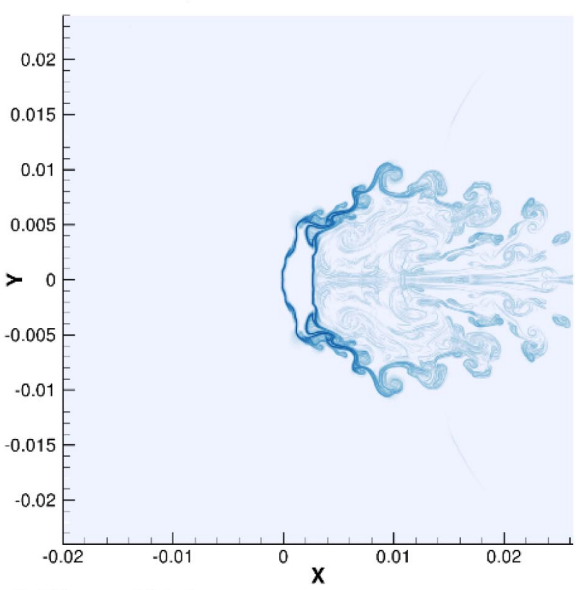

d) Time $=300.6 \mu \mathrm{s}$

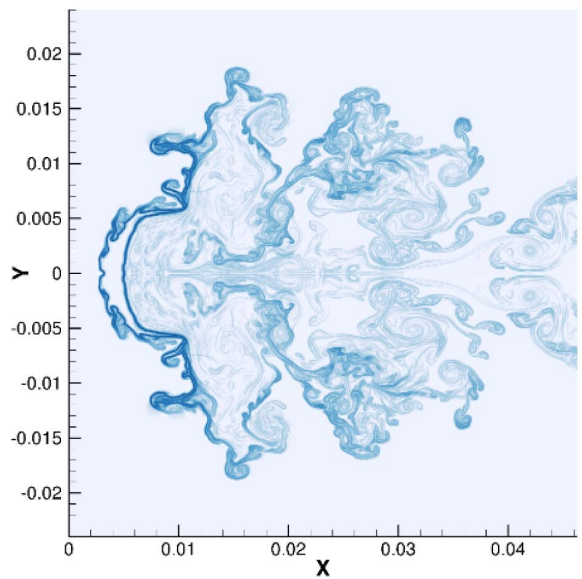

g) Time $=500.2 \mu \mathrm{s}$

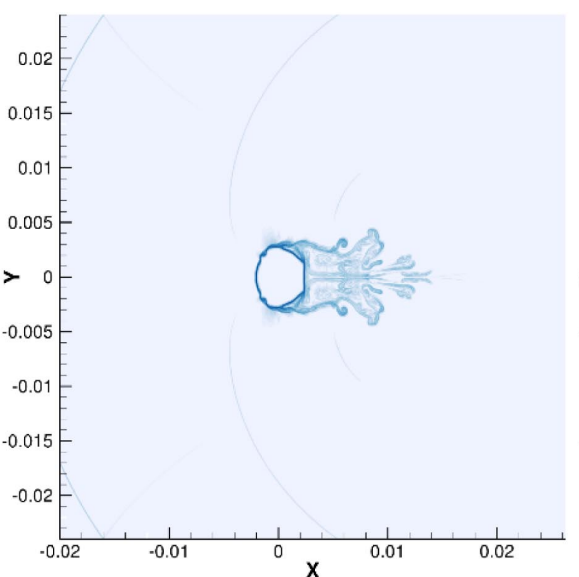

b) Time $=100.1 \mu \mathrm{s}$

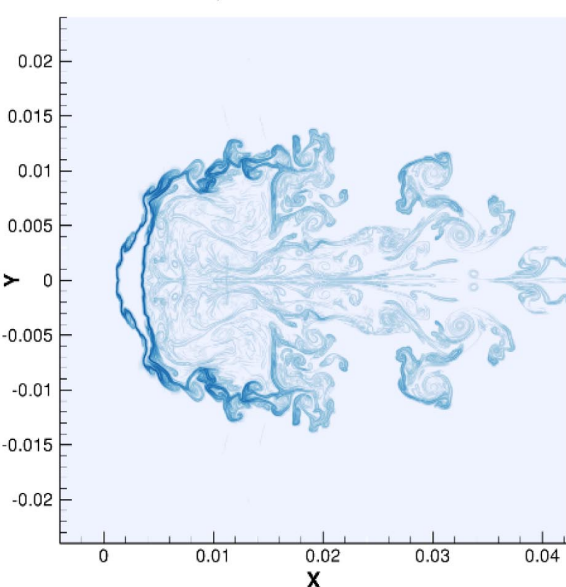

e) Time $=400.4 \mu \mathrm{s}$

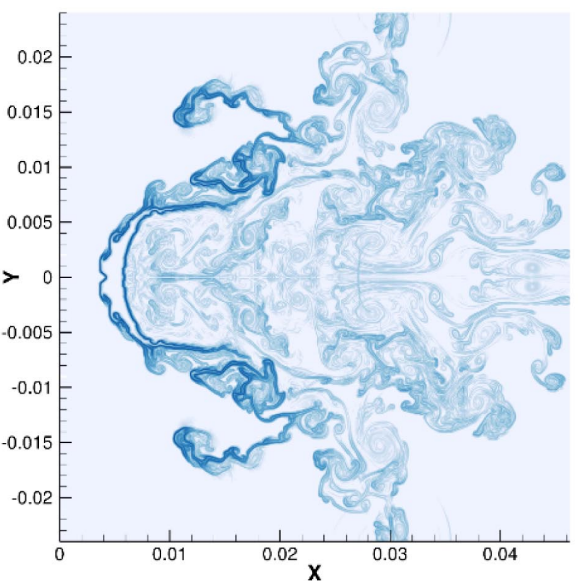

h) Time $=550.1 \mu \mathrm{s}$

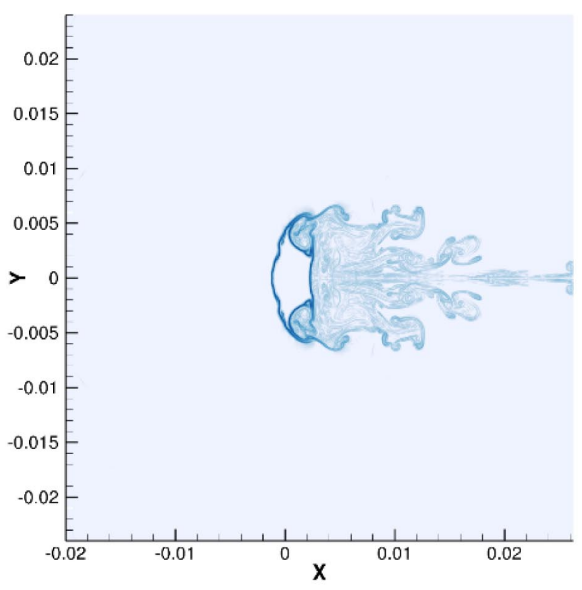

c) Time $=200.3 \mu \mathrm{s}$

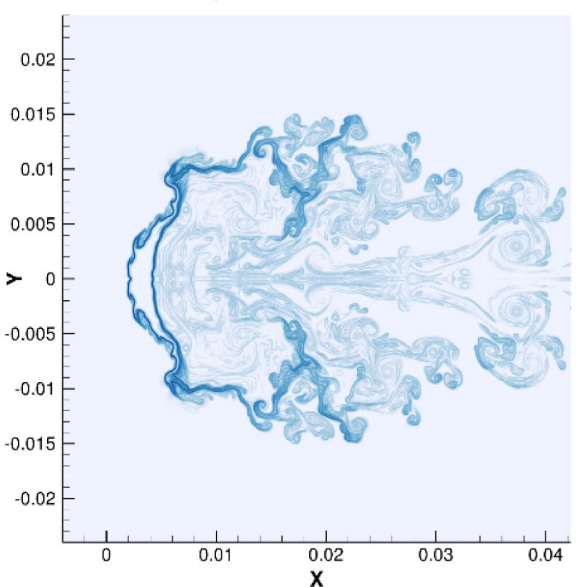

f) Time $=450.0 \mu \mathrm{s}$

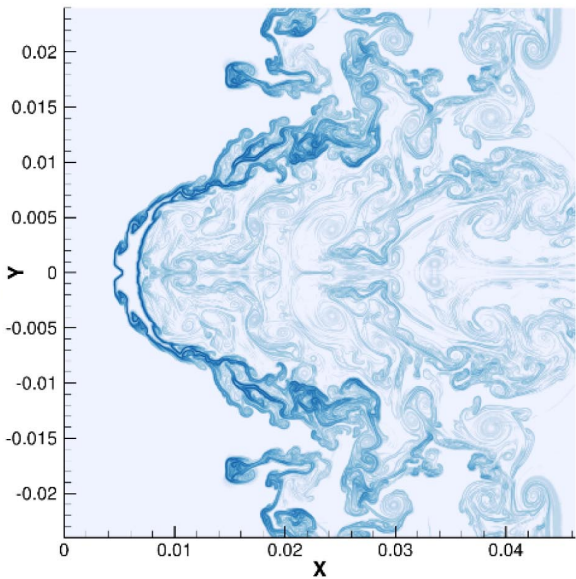

i) Time $=600.5 \mu \mathrm{s}$

Fig. 20 Evolution of the numerical schlieren plot during the stripping breakup of the water column [29]. Reprinted with permission from Elsevier.

Loh et al. [95] considered three selected problems (namely, a linear benchmark problem, instability waves on a free shear layer, and shock-vortex interactions) to further demonstrate CESE as a viable tool for computational aeroacoustics (CAA). The second-order CESE scheme yielded good numerical results with high resolution and low dispersion. Additionally, the authors also pointed out that the nonreflecting boundary condition, which plays an important role in CAA, is much simpler to implement in the CESE method than in traditional methods $[3,95]$. Yen et al. $[96,97]$ modified the Courantnumber-insensitive $\overline{\mathrm{CESE}}$ scheme [96] and the local time-stepping CESE scheme [97] to improve the numerical efficiency for realistic multidimensional CAA problems where large grid-size disparity is inevitable. A 3-D simulation was performed for the propagation of a Gaussian acoustic pulse and a vorticity wave embedded in a Mach 0.5 mean flow. The CESE results agreed well with the corresponding analytical solution in preserving both the form and amplitude of the waves [96]. A series of 3-D CESE simulations for the Helmholtz resonator problem were demonstrated in Ref. [97]. Excellent agreement between the linear acoustic theory and the CESE solution was achieved using just a second-order CESE scheme.

For CAA problems in practical engineering applications related to engines and aircraft, the CESE method has also been employed as a numerical tool. Loh and Zaman [98] developed an axisymmetric NS solver using the CESE method and conducted a numerical investigation to shed light upon an aeroacoustic resonance phenomenon often encountered by convergent-divergent nozzles under transonic conditions. Additionally, a 3-D CESE NS solver was implemented by Loh et al. [99] and applied to compute the screech noise generated 


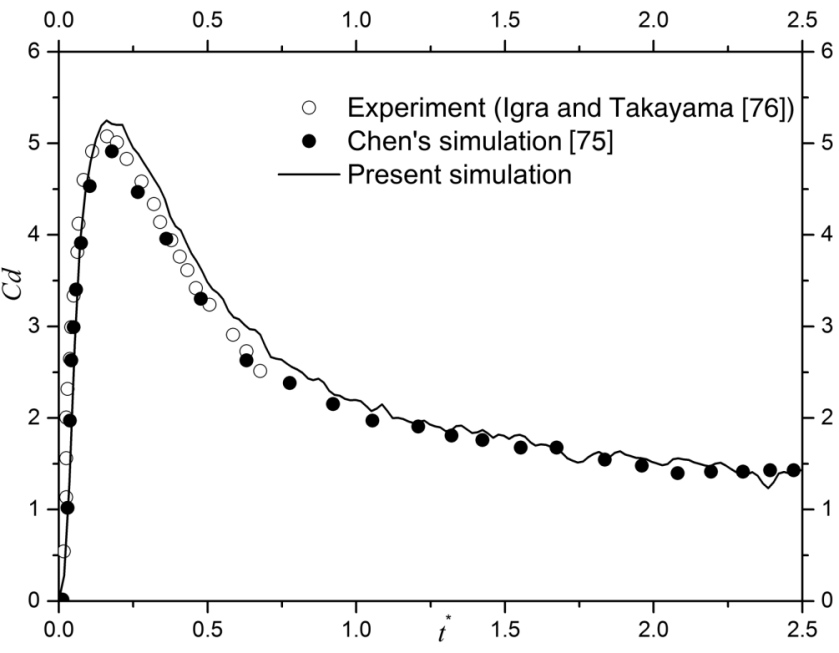

Fig. 21 Drag coefficient $C_{d}$ of water column impacted by shock. Dimensionless time $t^{*}$ defined as $t^{*}=t u_{g} / d$, where $u_{g}$ is postshock gas velocity and $d$ is diameter of water column [29]. Reprinted with permission from Elsevier.

by an underexpanded supersonic jet. Kim et al. [100] simulated the supersonic unsteady flow over an open cavity, which is considered as a mixing-enhancement and flame-holding approach in the scramjet engine concept. The CESE method was applied to solve 2-D NS equations, and it successfully captured the self-sustained oscillations in the supersonic cavity flows. The computed frequencies and amplitudes of the pressure oscillations compare favorably with the theoretical and experimental data. Cheng et al. [101] also studied subsonic and supersonic flows over open-cavity geometries. The generation and propagation of flow-induced acoustic waves were faithfully captured by an unsteady NS solver based on the CESE method. When compared with experimental and analytical data, satisfactory results were obtained for the oscillation frequency of the dominant mode.

\section{E. Computational Solid Dynamics}

In recent years, high-resolution discontinuity-capturing numerical methods devised for CFD problems have been considered as alternative approaches to solving nonlinear solid-dynamic problems with Eulerian formulations. Among these methods, the CESE method has attracted considerable attention from researchers in computational solid dynamics due to its simple logic, high accuracy, and ability to capture the behaviors of nonlinear waves.

Wang et al. [102] applied the CESE method to two high-velocity impact problems in elastic-plastic materials: the Taylor copperbar-impact problem and the penetration of a long rod (tungsten heavy alloy) into a steel target. In this numerical study, the CESE method was adopted to solve the Eulerian governing equations that describe the solid dynamics, and the level-set technique was used for tracing material interfaces. Chen et al. [103] extended the CESE solver in Ref. [102] to simulate high-velocity impact problems involving elastic-plastic flows, high strain rates, and spall fractures. Their simulation of aluminum plates colliding with stainless-steel plates showed excellent agreement with the experimental data.

By treating the density, velocity, and stress components as primitive unknowns, the governing equations of elastic waves in solids can be cast into a set of fully coupled first-order hyperbolic PDEs, including the conservation laws of mass and momentum as well as the rate-type constitutive relations for materials. Hence, the CESE method, which is suitable for hyperbolic systems, has been applied to simulate linear and nonlinear waves in elastic solids. Yu et al. [104] presented their CESE simulations of resonant standing waves arising from a time-harmonic external axial load and compression waves arising from a bimaterial collinear impact. Yang et al. [105] studied the propagation and reflection of extensional waves in an abruptly stopped elastic rod using the CESE method. Chen et al. [106] performed CESE simulations of planar-wave expansion from a point source in an anisotropic solid of cubic symmetry (gallium arsenide). Yang et al. [107] extended the CESE method to simulate stress waves in solids of hexagonal symmetry and studied wave propagation in a heterogeneous solid composed of three blocks of beryl with different lattice orientations. Using the CESE method as a numerical tool,

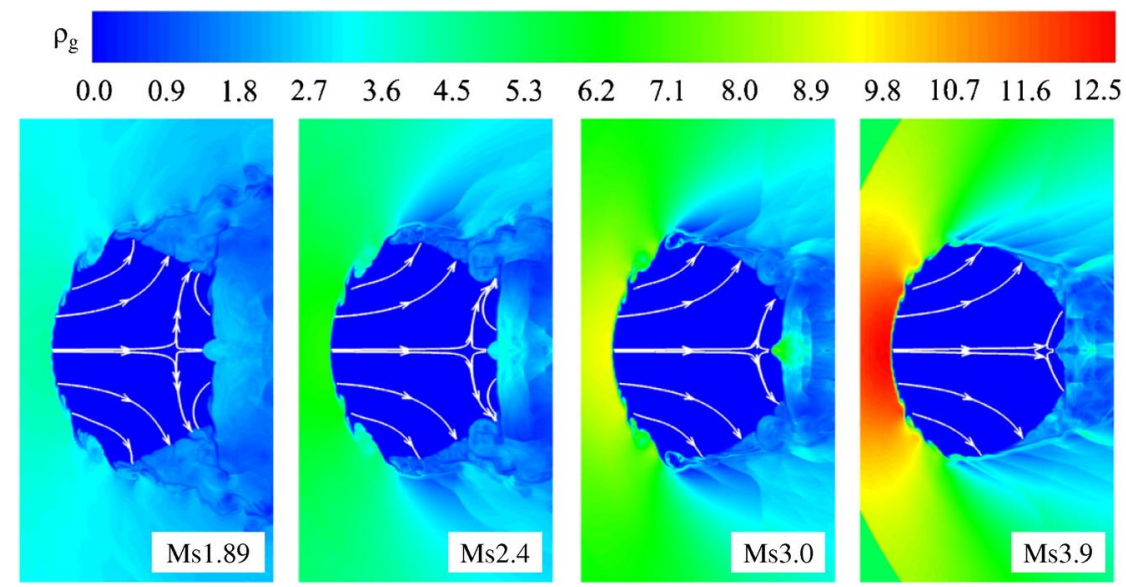

Fig. 22 Morphologies and internal flow patterns of a water droplet under shock impact with different shock Mach numbers $M_{s}$ [68]. Contours show density of air with units in kilograms per cubic meter. Reprinted with permission from AIAA.

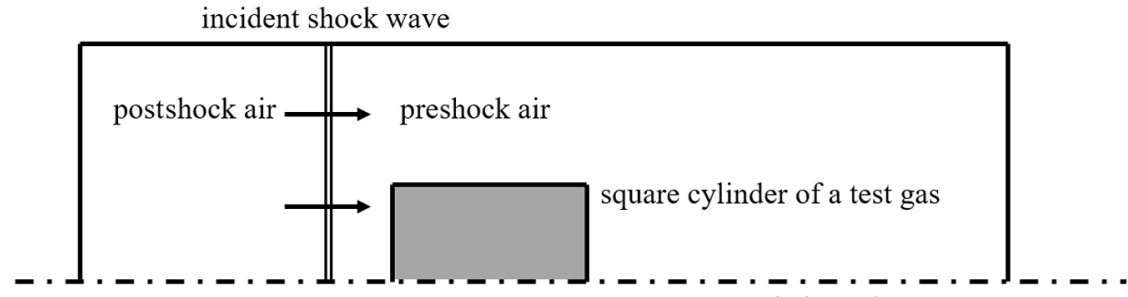

symmetric boundary

Fig. 23 A schematic of the initial setup for the interaction of a shock and a heavy gas inhomogeneity. 


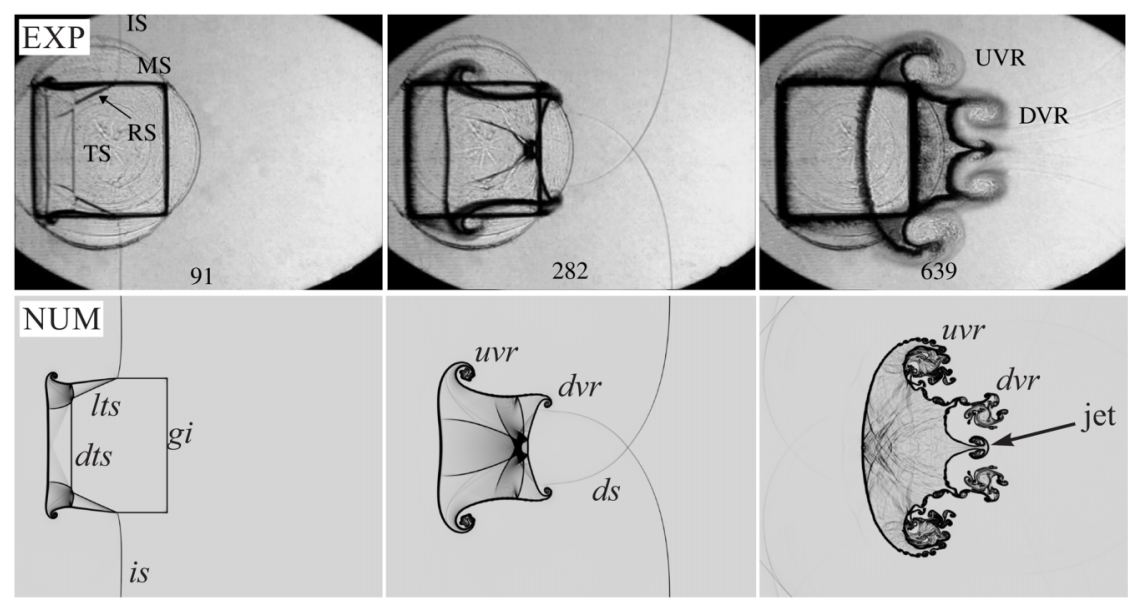

Fig. 24 Experimental images and numerical schlieren results for a shock-gas-cylinder interaction [69]. Reprinted with permission from AIP Publishing ( $i s=$ incident shock, $r s=$ refracted shock, $t s=$ transmitted shock, $m s=$ Mach stem, $g i=$ gas interface, $d t s=$ direct transmitted shock, $l t s=$ lateral transmitted shock, $d s=$ diffracted shock, $u v r=$ upstream vortex pair, $d v r=$ downstream vortex pair).

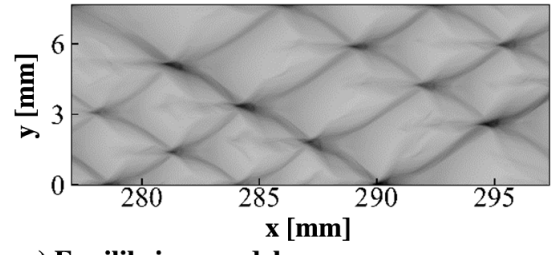

a) Equilibrium model

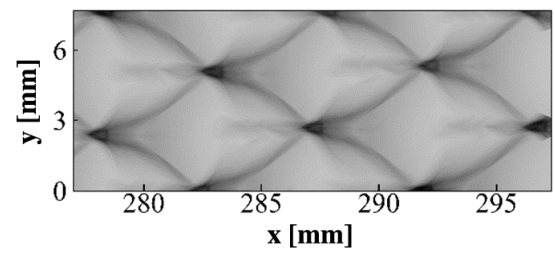

c) Park's two-temperature model

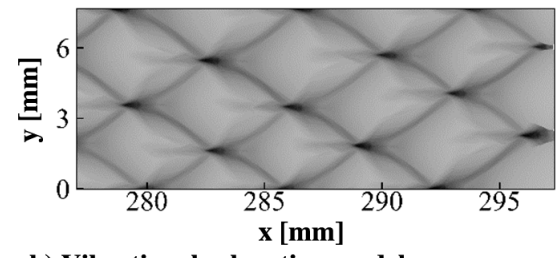

b) Vibrational relaxation model

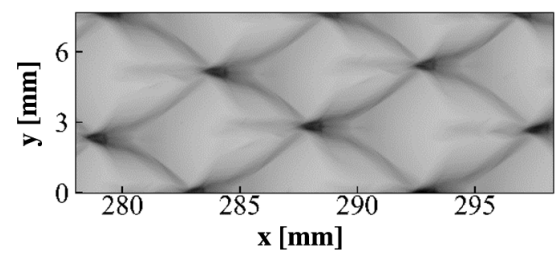

d) CVCV model

Fig. 25 Numerical soot foils in detonation simulations with four different physical models [ $\underline{83}$ ]. Reprinted with permission from Taylor and Francis.

Lowe et al. [108] conducted a comprehensive study of nonlinear longitudinal waves (including both weak and strong shocks, rarefactions, and contact discontinuities) in tapered elastic rods. Among these research works, the CESE simulations effectively captured waves in various solid materials and provided results consistent with the available analytical solutions.

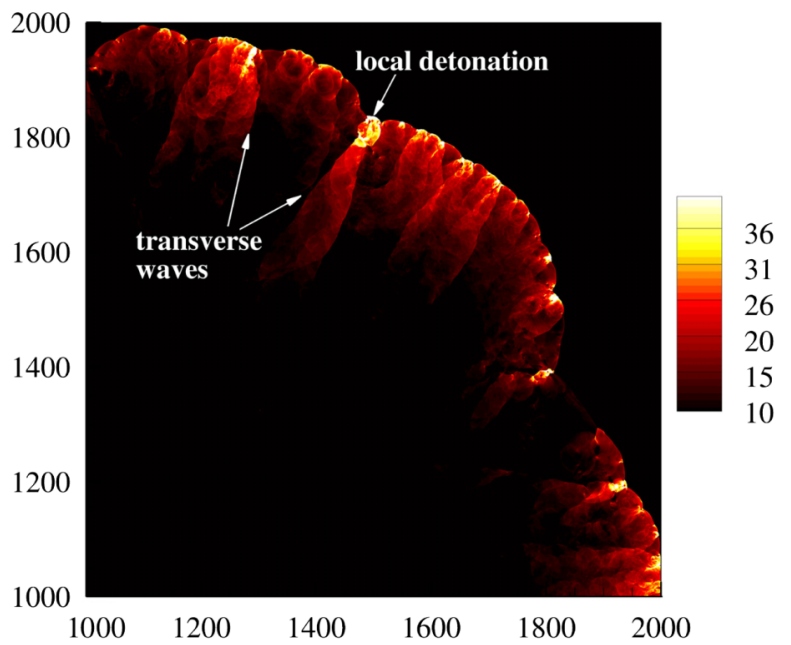

Fig. 26 Pressure contours (nondimensionalized by pressure of unburned reactant) in the 2-D simulation of detonation initiation [86]. Reprinted with permission from Cambridge University Press.

\section{F. Magnetohydrodynamics}

The magnetohydrodynamic equations, which combine the NS equations for fluid dynamics and the Maxwell equations for electromagnetics, can describe the plasma flows in aerospace applications and astrophysics. Because the computational MHD method is usually required to satisfy the divergence-free constraint for a magnetic field $\boldsymbol{B}$, some special treatments are incorporated into the MHD numerical schemes to enforce $\nabla \cdot \boldsymbol{B}=0$. Since the inception of the CESE method, researchers have applied this novel approach to the MHD equations to solve MHD problems accurately with a relatively simple algorithm.

Zhang et al. [109,110] studied MHD benchmark problems using the CESE method. Investigations of an MHD shock-tube problem and an MHD-vortex problem demonstrated that the original CESE method can be directly used to solve the MHD equations.

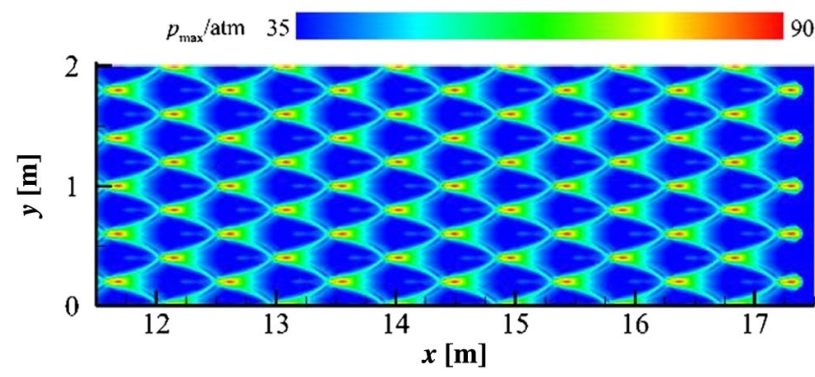

Fig. 27 Traces of maximum pressure in an aluminum-air detonation [92]. Reprinted with permission from Elsevier. 
According to the numerical comparisons reported in Refs. [109,110], the CESE results without additional treatments for $\nabla \cdot \boldsymbol{B}=0 \mathrm{com}$ pared favorably with previously reported reference solutions. This finding mainly comes from the observation of the numerical results, and the reason is not clear yet. Actually, in Refs. [109,110], comparisons were performed between the baseline CESE scheme and the CESE scheme in conjunction with a special treatment to maintain $\nabla \cdot \boldsymbol{B}=0$, but no obvious difference was observed.

Feng et al. [111] developed a numerical platform to investigate solar-interplanetary physics and space weather. The CESE method was adopted to solve 3-D MHD equations, and adaptive mesh refinement was used to better resolve flow features that have spatial scales many orders of magnitude smaller than the vast size of solarinterplanetary space. This CESE-based MHD-simulation approach was validated through the numerical study of solar corona, solar wind, and comparison with observation data. Recently, Yang et al. [34,39] extended this CESE MHD solver to a high-order version [39] as well as an upwind version [34] using the methodology of Shen et al. [26], Shen and Wen [27], and Shen et al. [37]. Numerical tests including MHD-vortex and MHD-blast-wave problems indicated that both the high-order and the upwind extensions can improve the accuracy of CESE results. Furthermore, a new strategy to keep the magnetic field fundamentally divergence free was proposed in Ref. [39], which uses the advantage of the CESE method that the spatial derivatives of the magnetic field are treated as marching variables in the algorithm.

\section{G. Computational Electromagnetics}

Numerical techniques such as the finite difference, finite volume, and finite element methods have been applied to time-domain simulations of electromagnetic fields and waves by solving Maxwell's equations. Nevertheless, recent development of the CESE method provides an alternative framework for computational electromagnetics.

Wang et al. [112] used the CESE method to solve the scattering field of transverse magnetic waves due to a perfect conducting circular cylinder. The computed results of the electric and magnetic intensity distributions on the cylinder surface, the surface current, and the radar cross section showed good agreement with the analytical solutions. Sessions and Winans [113] developed a generalized Maxwell solver based on the Courant-number-insensitive CESE scheme [6]. In their work, electromagnetic wave propagation in nondispersive and dispersive materials was studied numerically. The second-order CESE method was shown to be accurate on highly nonuniform meshes and performed well for problems involving highly discontinuous material properties.

\section{H. Hydraulic Engineering}

In hydraulic engineering, free-surface gravity flows in rivers and reservoirs can be approximately described by the shallow-water equations, which form a nonlinear hyperbolic system of conservation laws. The classical shallow-water equations are also known as the Saint-Venant equations. Although multiple assumptions have been made in the derivation of the shallow-water equations, their solutions remain challenging for numerical methods.

Molls and Molls [114] applied the CESE method to the 1-D and 2-D Saint-Venant equations and simulated a 1-D idealized dam break, a 1-D hydraulic jump in a straight rectangular channel, and a 2-D oblique hydraulic jump. The CESE results were compared with experimental data, analytical solutions, and numerical results obtained by conventional high-resolution schemes. These comparisons indicated that the CESE method is simple, robust, and accurate for hydraulic applications. Saleem et al. [115] applied the CESE method to a set of extended shallow-water equations incorporating temperature gradients and variable bottom topography. Extensive numerical simulations of dambreak problems and perturbation problems verified the high accuracy of the CESE method.

\section{Other Applications}

In addition to the research fields covered by Secs. VI.A-VI.H, many other scientific and engineering problems also benefit from the capabilities of the CESE method. The following are some examples. Lim et al. [116] and Yao et al. [117] studied chromatographic adsorption problems in chemical engineering using the CESE method. Chou and Yang [118] solved the non-Fourier heat conduction equations by using the $\overline{C E S E}$ method to simulate the behavior of thermal waves in ultrafast heat conduction processes. To simulate the non-Fourier heat conduction in solids at low temperatures, Qamar and Ashraf [119] employed the CESE method to solve the hyperbolic system derived by taking moments of the Boltzmann-Peierls kinetic equation for phonon transport. Noor and Qamar [120] implemented the CESE method for simulating the batch crystallization process with fines dissolution. Nisar et al. [121] performed numerical simulations of the charge transport in semiconductor devices by solving a hydrodynamical model with the CESE method. Rehman et al. [122] solved a dusty-gas-flow model using the upwind HLLC-CESE scheme proposed by Shen et al. [26].

\section{Conclusions}

The CESE method is an explicit one-step compact finitevolume-type numerical method that provides an alternative approach to accurate, robust, and efficient computations of scientific and engineering problems. From a historical perspective, this paper has reviewed the development of the CESE method since the 1990s and summarized the remarkable improvements and extensions of the CESE method in the last five years. A variety of applications of the method have been presented, with emphasis on its numerical performance under different scenarios.

The essential ingredients in the CESE method include the following:

1) Spatial derivatives of physical quantities are stored and updated as independent unknowns.

2) A staggered space-time mesh is employed.

3) The interior structure within each solution element is built with the Taylor expansion.

4) The time-marching approach is based on the Cauchy-Kowalewski procedure.

Individually, none of these considerations is new, but the CESE method combines them together. As a result, the CESE method possesses a low numerical dissipation and a high compactness. Numerical examples, including benchmark cases and applications, indicate that the CESE method has a high resolution of shock waves, shear layers, multimaterial interfaces, fine structures, and small disturbances. Therefore, the CESE method demonstrates good performances in the numerical investigations of wave-propagation problems, such as detonations, aeroacoustics, shock-induced interfacial instabilities, and shock-bubble/droplet interactions.

In the future, attention will be paid to the extension of CESE schemes to implicit time-stepping schemes.

\section{Acknowledgments}

This research is supported by the Hong Kong Research Grants Council (no. 152151/16E, no. 152041/18E, and no. 152065/19E) and the National Natural Science Foundation of China (no. 11772284). The authors would like to thank Hua Shen for useful discussions on the conservation element and solution element method. The authors would also like to thank Jiaao Hao for providing finite volume simulation results for the "Mach 3 step" case.

\section{References}

[1] Chang, S.-C., and To, W.-M., "A New Numerical Framework for Solving Conservation Laws-The Method of Space-Time Conservation Element and Solution Element," NASA TM-104495, 1991.

[2] Chang, S.-C., "The Method of Space-Time Conservation Element and Solution Element-A New Approach for Solving the Navier-Stokes and Euler Equations," Journal of Computational Physics, Vol. 119, No. 2, 1995, pp. 295-324.

https://doi.org/10.1006/jcph.1995.1137

[3] Chang, S.-C., Loh, C. Y., Yu, S.-T., Himansu, A., Wang, X.-Y., and Jorgenson, P. C. E., "Robust and Simple Non-Reflecting Boundary Conditions for the Space-Time Conservation Element and Solution 
Element Method," 13th Computational Fluid Dynamics Conference, AIAA Paper 1997-2077, 1997.

https://doi.org/10.2514/6.1997-2077

[4] Chang, S.-C., Wang, X.-Y., and Chow, C.-Y., "The Space-Time Conservation Element and Solution Element Method: A New HighResolution and Genuinely Multidimensional Paradigm for Solving Conservation Laws," Journal of Computational Physics, Vol. 156, No. 1, 1999, pp. 89-136. https://doi.org/10.1006/jcph.1999.6354

[5] Chang, S.-C., Wang, X.-Y., and To, W.-M., "Application of the Space-Time Conservation Element and Solution Element Method to One-Dimensional Convection-Diffusion Problems," Journal of Computational Physics, Vol. 165, No. 1, 2000, pp. 189-215. https://doi.org/10.1006/jcph.2000.6610

[6] Chang, S.-C., "Courant Number Insensitive CE/SE Schemes," 38th AIAA/ASME/SAE/ASEE Joint Propulsion Conference and Exhibit, AIAA Paper 2002-3890, 2002. https://doi.org/10.2514/6.2002-3890

[7] Chang, S.-C., and Wang, X.-Y., "Multi-Dimensional Courant Number Insensitive CE/SE Euler Solvers for Applications Involving Highly Nonuniform Meshes," 39th AIAA/ASME/SAE/ASEE Joint Propulsion Conference and Exhibit, AIAA Paper 2003-5285, 2003. https://doi.org/10.2514/6.2003-5285

[8] Chang, S.-C., "Courant Number and Mach Number Insensitive CE/SE Euler Solvers," 41st AIAA/ASME/SAE/ASEE Joint Propulsion Conference and Exhibit, AIAA Paper 2005-4355, 2005. https://doi.org/10.2514/6.2005-4355

[9] Wang, X.-Y., Chang, S.-C., Kao, K.-H., Jorgenson, P., and Chow, C.-Y., "A Non-Splitting Unstructured-Triangular-Mesh Euler Solver Based on the Method of Space-Time Conservation Element and Solution Element," 16th International Conference on Numerical Methods in Fluid Dynamics, Springer, Berlin, 1998, pp. 97-102. https://doi.org/10.1007/BFb0106568

[10] Wang, X.-Y., and Chang, S.-C., "A 2-D Non-Splitting Unstructured Triangular Mesh Euler Solver Based on the Space-Time Conservation Element and Solution Element Method," Computational Fluid Dynamics Journal, Vol. 8, No. 2, 1999, pp. 309-325.

[11] Wang, X.-Y., and Chang, S.-C., "A 3-D Non-Splitting Structured/ Unstructured Euler Solver Based on the Space-Time Conservation Element and Solution Element Method," 14th Computational Fluid Dynamics Conference, AIAA Paper 1999-3278, 1999, pp. 256-268. https://doi.org/10.2514/6.1999-3278

[12] Zhang, Z.-C., Yu, S. T. J., and Chang, S.-C., "A Space-Time Conservation Element and Solution Element Method for Solving the Two- and Three-Dimensional Unsteady Euler Equations Using Quadrilateral and Hexahedral Meshes," Journal of Computational Physics, Vol. 175, No. 1, 2002, pp. 168-199. https://doi.org/10.1006/jcph.2001.6934

[13] Guo, Y., Hsu, A. T., Wu, J., Yang, Z., and Oyediran, A., "Extension of CE/SE Method to 2-D Viscous Flows," Computers and Fluids, Vol. 33, No. 10, 2004, pp. 1349-1361. https://doi.org/10.1016/i.compfluid.2003.10.005

[14] Chang, C.-L., "Time-Accurate, Unstructured-Mesh Navier-Stokes Computations with the Space-Time CESE Method," 42nd AIAA/ ASME/SAE/ASEE Joint Propulsion Conference and Exhibit, AIAA Paper 2006-4780, 2006.

https://doi.org/10.2514/6.2006-4780

[15] Chang, S.-C., Wu, Y., Yang, V., and Wang, X.-Y., "Local TimeStepping Procedures for the Space-Time Conservation Element and Solution Element Method," International Journal of Computational Fluid Dynamics, Vol. 19, No. 5, 2005, pp. 359-380. https://doi.org/10.1080/10618560500092610

[16] Chang, S.-C., "The a(4) Scheme-A High Order Neutrally Stable CESE Solver," 43rd AIAA/ASME/SAE/ASEE Joint Propulsion Conference and Exhibit, AIAA Paper 2007-5820, 2007. https://doi.org/10.2514/6.2007-5820

[17] Chang, S.-C., "On Space-Time Inversion Invariance and Its Relation to Non-Dissipatedness of a CESE Core Scheme," 42nd AIAA/ASME/ SAE/ASEE Joint Propulsion Conference and Exhibit, AIAA Paper 2006-4779, July 2006. https://doi.org/10.2514/6.2006-4779

[18] Yang, D., Yu, S.-T., and Zhao, J., "Convergence and Error Bound Analysis for the Space-Time CESE Method," Numerical Methods for Partial Differential Equations, Vol. 17, No. 1, 2001, pp. 64-78. https://doi.org/10.1002/1098-2426(200101)17:1<64::AID-NUM5>3 .0.CO;2-6

[19] Venkatachari, B. S., Cheng, G. C., Soni, B. K., and Chang, S.-C., "Validation and Verification of Courant Number Insensitive CE/SE Method for Transient Viscous Flow Simulations," Mathematics and
Computers in Simulation, Vol. 78, Nos. 5-6, 2008, pp. 653-670. https://doi.org/10.1016/j.matcom.2008.04.007

[20] Cheng, G. C., Venkatachari, B. S., Chang, C.-L., and Chang, S.-C., "Comparative Study of Different Numerical Approaches in SpaceTime CESE Framework for High-Fidelity Flow Simulations," Computers and Fluids, Vol. 45, No. 1, 2011, pp. 47-54. https://doi.org/10.1016/j.compfluid.2011.01.030

[21] Jiang, C., Cui, S., and Feng, X., "Solving the Euler and Navier-Stokes Equations by the AMR-CESE Method," Computers and Fluids, Vol. 54, Jan. 2012, pp. 105-117. https://doi.org/10.1016/i.compfluid.2011.10.006

[22] Shen, H., Liu, K., and Zhang, D., "A Local Space-Time Conservation Scheme and Its Application in Shock Wave Propagation," Applied Mathematics and Computation, Vol. 219, No. 4, 2012, pp. 1958-1974. https://doi.org/10.1016/j.amc.2012.08.038

[23] Liu, K., Wang, J., Wang, G., Chen, Q., Fu, Z., and Wu, S., "A Review on the CE/SE Method," Advances in Mechanics, Vol. 41, No. 4, 2011, pp. 447-461. https://doi.org/10.6052/1000-0992-2011-4-1xjzJ2010-136

[24] Cook, G. O., Jr., Zhang, Z., and Im, K.-S., "Applications of the CESE Method in LS-DYNA," 21st AIAA Computational Fluid Dynamics Conference, AIAA Paper 2013-3070, 2013. https://doi.org/10.2514/6.2013-3070

[25] Wang, X.-Y., "A Summary of the Space-Time Conservation Element and Solution Element (CESE) Method," NASA TM-2015-218743, 2015.

[26] Shen, H., Wen, C.-Y., and Zhang, D.-L., "A Characteristic Space-Time Conservation Element and Solution Element Method for Conservation Laws," Journal of Computational Physics, Vol. 288, May 2015, pp. 101-118. https://doi.org/10.1016/j.jcp.2015.02.018

[27] Shen, H., and Wen, C.-Y., "A Characteristic Space-Time Conservation Element and Solution Element Method for Conservation Laws II. Multidimensional Extension," Journal of Computational Physics, Vol. 305, Jan. 2016, pp. 775-792. https://doi.org/10.1016/j.jcp.2015.11.017

[28] Shen, H., and Wen, C.-Y., "A Characteristic Space-Time CE/SE Method for Shock Capturing," 30th International Symposium on Shock Waves, Vol. 2, Springer, Switzerland, Cham, 2017, pp. 1495-1550. https://doi.org/10.1007/978-3-319-44866-4_123

[29] Shen, H., Wen, C.-Y., Parsani, M., and Shu, C.-W., "MaximumPrinciple-Satisfying Space-Time Conservation Element and Solution Element Scheme Applied to Compressible Multifluids," Journal of Computational Physics, Vol. 330, Feb. 2017, pp. 668-692. https://doi.org/10.1016/j.jcp.2016.10.036

[30] Shen, H., and Parsani, M., "Positivity-Preserving CESE Schemes for Solving the Compressible Euler and Navier-Stokes Equations on Hybrid Unstructured Meshes," Computer Physics Communications, Vol. 232, Nov. 2018, pp. 165-176. https://doi.org/10.1016/j.cpc.2018.05.011

[31] Harten, A., Lax, P. D., and van Leer, B., "On Upstream Differencing and Godunov-Type Schemes for Hyperbolic Conservation Laws," SIAM Review, Vol. 25, No. 1, 1983, pp. 35-61. https://doi.org/10.1137/1025002

[32] Toro, E. F., Spruce, M., and Speares, W., "Restoration of the Contact Surface in the HLL-Riemann Solver," Shock Waves, Vol. 4, No. 1, 1994, pp. 25-34. https://doi.org/10.1007/BF01414629

[33] Roe, P. L., "Approximate Riemann Solvers, Parameter Vectors, and Difference Schemes," Journal of Computational Physics, Vol. 43, No. 2, 1981, pp. 357-372. https://doi.org/10.1016/0021-9991(81)90128-5

[34] Yang, Y., Feng, X.-S., and Jiang, C.-W., "An Upwind CESE Scheme for 2-D and 3-D MHD Numerical Simulation in General Curvilinear Coordinates," Journal of Computational Physics, Vol. 371, Oct. 2018, pp. 850-869. https://doi.org/10.1016/j.jcp.2018.05.014

[35] Fu, Z., and Liu, K.-X., "An Improved Two-Dimensional Unstructured CE/SE Scheme for Capturing Shock Waves," Chinese Physics B, Vol. 21, No. 4, 2012, Paper 040202. https://doi.org/10.1088/1674-1056/21/4/040202

[36] Shen, H., and Parsani, M., "A Rezoning-Free CESE Scheme for Solving the Compressible Euler Equations on Moving Unstructured Meshes," Journal of Computational Physics, Vol. 397, Nov. 2019, Paper 108858. https://doi.org/10.1016/j.jcp.2019.108858

[37] Shen, H., Wen, C.-Y., Liu, K.-X., and Zhang, D.-L., "Robust HighOrder Space-Time Conservative Schemes for Solving Conservation Laws on Hybrid Meshes," Journal of Computational Physics, Vol. 281, 
Jan. 2015, pp. 375-402.

https://doi.org/10.1016/j.jcp.2014.10.023

[38] Liu, K.-X., and Wang, J.-T., "Analysis of High Accuracy ConservationElement and Solution-Element Schemes," Chinese Physics Letter, Vol. 21, No. 11, 2004, pp. 2085-2088. https://doi.org/10.1088/0256-307X/21/11/004

[39] Yang, Y., Feng, X.-S., and Jiang, C.-W., "A High-Order CESE Scheme with a New Divergence-Free Method for MHD Numerical Simulation," Journal of Computational Physics, Vol. 349, Nov. 2017, pp. 561-581. https://doi.org/10.1016/j.jcp.2017.08.019

[40] Bilyeu, D. L., Yu, S.-T. J., Chen, Y.-Y., and Cambier, J.-L., "A TwoDimensional Fourth-Order Unstructured-Meshed Euler Solver Based on the CESE Method," Journal of Computational Physics, Vol. 257, Jan. 2014, pp. 981-999. https://doi.org/10.1016/j.jcp.2013.09.044

[41] Chang, S.-C., "A New Approach for Constructing Highly Stable High Order CESE Schemes," 48th AIAA Aerospace Science Meeting, AIAA Paper 2010-543, 2010. https://doi.org/10.2514/6.2010-543

[42] Sod, G. A., "A Survey of Several Finite Difference Methods for Systems of Non-Linear Hyperbolic Conservation Laws," Journal of Computational Physics, Vol. 27, No. 1, 1978, pp. 1-31. https://doi.org/10.1016/0021-9991(78)90023-2

[43] Woodward, P., and Colella, P., "The Numerical Simulation of Two-Dimensional Fluid Flow with Strong Shocks," Journal of Computational Physics, Vol. 54, No. 1, 1984, pp. 115-173. https://doi.org/10.1016/0021-9991(84)90142-6

[44] Hao, J., Wang, J., and Lee, C., "Numerical Simulation of High-Enthalpy Double-Cone Flows," AIAA Journal, Vol. 55, No. 7, 2017, pp. 2471-2475. https://doi.org/10.2514/1.J055746

[45] Fedorov, A., and Tumin, A., "Evolution of Disturbances in Entropy Layer on Blunted Plate in Supersonic Flow," AIAA Journal, Vol. 42, No. 1, 2004, pp. 89-94. https://doi.org/10.2514/1.9033

[46] Tseng, T. I., and Yang, R. J., "Simulation of the Mach Reflection in Supersonic Flows by the CE/SE Method," Shock Waves, Vol. 14, No. 4, 2005, pp. 307-311. https://doi.org/10.1007/s00193-005-0255-8

[47] Chang, I-S., Chang, C.-L., and Chang, S.-C., "Unsteady Navier-Stokes Rocket Nozzle Flows," 41 st AIAA/ASME/SAE/ASEE Joint Propulsion Conference and Exhibit, AIAA Paper 2005-4353, 2005. https://doi.org/10.2514/6.2005-4353

[48] Chang, C.-L., Venkatachari, B. S., and Cheng, G. C., "Effect of Counterflow Jet on a Supersonic Reentry Capsule," 42nd AIAA/ASME/SAE/ASEE Joint Propulsion Conference and Exhibit, AIAA Paper 2006-4776, 2006. https://doi.org/10.2514/6.2006-4776

[49] Venkatachari, B. S., Ito, Y., Cheng, G., and Chang, C.-L., "Numerical Investigation of the Interaction of Counterflowing Jets and Supersonic Capsule Flows," 42nd AIAA Thermophysics Conference, AIAA Paper 2011-4030, 2011. https://doi.org/10.2514/6.2011-4030

[50] Chang, C.-L., and Choudhari, M. M., "Hypersonic Viscous Flow over Large Roughness Elements," Theoretical and Computational Fluid Dynamics, Vol. 25, Nos. 1-4, 2011, pp. 85-104. https://doi.org/10.1007/s00162-010-0191-9

[51] Wen, C.-Y., Massimi, H. S., and Shen, H., "Extension of CE/SE Method to Non-Equilibrium Dissociating Flows," Journal of Computational Physics, Vol. 356, March 2018, pp. 240-260. https://doi.org/10.1016/j.jcp.2017.12.005

[52] Shen, H., Wen, C.-Y., and Massimi, H. S., "Application of CE/SE Method to Study Hypersonic Non-Equilibrium Flows over Spheres," 19th AIAA International Space Planes and Hypersonic Systems and Technologies Conference, AIAA Paper 2014-2509, June 2014. https://doi.org/10.2514/6.2014-2509

[53] Massimi, H. S., Shen, H., and Wen, C.-Y., "Study of Hypersonic Dissociating Flows over Spheres Using the Space-Time CE/SE Method," 30th International Symposium on Shock Waves, Vol. 1, Springer, Switzerland, Cham, 2017, pp. 145-148. https://doi.org/10.1007/978-3-319-46213-4_23

[54] Knab, O., Fruehauf, H.-H., and Messerschmid, E. W., "Theory and Validation of the Physically Consistent Coupled VibrationChemistry-Vibration Model," Journal of Thermophysics and Heat Transfer, Vol. 9, No. 2, 1995, pp. 219-226. https://doi.org/10.2514/3.649

[55] Wen, C.-Y., and Hornung, H. G., "Non-Equilibrium Dissociating Flow over Spheres," Journal of Fluid Mechanics, Vol. 299, Sept.
1995, pp. 389-405.

https://doi.org/10.1017/S0022112095003545

[56] Massimi, H. S., Shen, H., and Wen, C.-Y., "Numerical Simulation of Ionized Hypersonic Flows using the Space-Time CESE Method," 20th AIAA International Space Planes and Hypersonic Systems and Technologies Conference, AIAA Paper 2015-3636, July 2015. https://doi.org/10.2514/6.2015-3636

[57] Candler, G. V., and MacCormack, R. W., "Computation of Weakly Ionized Hypersonic Flows in Thermochemical Nonequilibrium," Journal of Thermophysics and Heat Transfer, Vol. 5, No. 3, 1991, pp. 266-273.

https://doi.org/10.2514/3.260

[58] Qamar, S., Ahmed, M., and Ali, I., "The Space-Time CE/SE Method for Solving Reduced Two-Fluid Flow Model," Communication in Computational Physics, Vol. 12, No. 4, 2012, pp. 1070-1095. https://doi.org/10.4208/cicp.210211.011111a

[59] Kreeft, J. J., and Koren, B., "A New Formulation of Kapila's FiveEquation Model for Compressible Two-Fluid Flow and Its Numerical Treatment," Journal of Computational Physics, Vol. 229, No. 18, 2010, pp. 6220-6242. https://doi.org/10.1016/j.jcp.2010.04.025

[60] Nessyahu, H., and Tadmor, E., "Non-Oscillatory Central Differencing for Hyperbolic Conservation Laws," Journal of Computational Physics, Vol. 87, No. 2, 1990, pp. 408-463. https://doi.org/10.1016/0021-9991(90)90260-8

[61] Qamar, S., and Ahmed, M., "A High Order Kinetic Flux-Vector Splitting Method for the Reduced Five-Equation Model of Compressible Two-Fluid Flows," Journal of Computational Physics, Vol. 228, No. 24, 2009, pp. 9059-9078. https://doi.org/10.1016/j.jcp.2009.09.010

[62] Fu, Z., Liu, K.-X., and Luo, N., "Simulation of Shock-Induced Instability Using an Essentially Conservative Adaptive CE/SE Method," Chinese Physics B, Vol. 23, No. 2, 2014, Paper 020202. https://doi.org/10.1088/1674-1056/23/2/020202

[63] Collins, B. D., and Jacobs, J. W., "PLIF Flow Visualization and Measurements of the Richtmyer-Meshkov Instability of an Air/SF6 Interface," Journal of Fluid Mechanics, Vol. 464, Aug. 2002, pp. 113-136. https://doi.org/10.1017/S0022112002008844

[64] Zhai, Z., Li, W., Si, T., Luo, X., Yang, J., and Lu, X., "Refraction of Cylindrical Converging Shock Wave at an Air/Helium Gaseous Interface," Physics of Fluids, Vol. 29, No. 1, 2017, Paper 016102. https://doi.org/10.1063/1.4973825

[65] Zhou, Z., Ding, J., Zhai, Z., Cheng, W., and Luo, X., "Mode Coupling in Converging Richtmyer-Meshkov Instability of Dual-Mode Interface," Acta Mechanica Sinica, Vol. 36, April 2020, pp. 356-366. https://doi.org/10.1007/s10409-019-00917-3

[66] Zhai, Z., Zhang, F., Zhou, Z., Ding, J., and Wen, C.-Y., "Numerical Study on Rayleigh-Taylor Effect on Cylindrically Converging Richtmyer-Meshkov Instability," Science China Physics, Mechanics and Astronomy, Vol. 62, No. 12, 2019, pp. 124712-124721. https://doi.org/10.1007/s11433-019-9441-4

[67] Liang, Y., Zhai, Z., Luo, X., and Wen, C.-Y., "Interfacial Instability at a Heavy/Light Interface Induced by Rarefaction Waves," Journal of Fluid Mechanics, Vol. 885, Feb. 2020, pp. A42-A60. https://doi.org/10.1017/jfm.2019.1025

[68] Guan, B., Liu, Y., Wen, C.-Y., and Shen, H., "Numerical Study on Liquid Droplet Internal Flow Under Shock Impact," AIAA Journal, Vol. 56, No. 9, 2018, pp. 3382-3387. https://doi.org/10.2514/1.J057134

[69] Fan, E, Guan, B., Wen, C.-Y., and Shen, H., "Numerical Study on Jet Formation of Simple-Geometry Heavy Gas Inhomogeneities," Physics of Fluids, Vol. 31, No. 2, 2019, Paper 026103. https://doi.org/10.1063/1.5083636

[70] Shyue, K.-M., "An Efficient Shock-Capturing Algorithm for Compressible Multicomponent Problems," Journal of Computational Physics, Vol. 142, No. 1, 1998, pp. 208-242. https://doi.org/10.1006/jcph.1998.5930

[71] Saurel, R., and Abgrall, R., "A Simple Method for Compressible Multifluid Flows," SIAM Journal on Scientific Computing, Vol. 21, No. 3, 1999, pp. 1115-1145. https://doi.org/10.1137/S1064827597323749

[72] Zhang, X., and Shu, C.-W., "On Maximum-Principle-Satisfying High Order Schemes for Scalar Conservation Laws," Journal of Computational Physics, Vol. 229, No. 9, 2010, pp. 3091-3120. https://doi.org/10.1016/j.jcp.2009.12.030

[73] Ranjan, D., Oakley, J., and Bonazza, R., "Shock-Bubble Interactions," Annual Review of Fluid Mechanics, Vol. 43, Jan. 2011, pp. 117-140. https://doi.org/10.1146/annurev-fluid-122109-160744 
[74] Terashima, H., and Tryggvason, G., "A Front-Tracking/Ghost-Fluid Method for Fluid Interfaces in Compressible Flows," Journal of Computational Physics, Vol. 228, No. 11, 2009, pp. 4012-4037. https://doi.org/10.1016/j.jcp.2009.02.023

[75] Chen, H., "Two-Dimensional Simulation of Stripping Breakup of a Water Droplet," AIAA Journal, Vol. 46, No. 5, 2008, pp. 1135-1143. https://doi.org/10.2514/1.31286

[76] Igra, D., and Takayama, K., "A Study of Shock Wave Loading on a Cylindrical Water Column," Reports of the Institute of Fluid Science, Tohoku University, Vol. 13, Tohoku Univ., Sendai, Japan, 2001, pp. 19-36.

[77] Luo, X., Wang, M., Si, T., and Zhai, Z., "On the Interaction of a Planar Shock with an SF6 Polygon," Journal of Fluid Mechanics, Vol. 773, Jan. 2015, pp. 366-394. https://doi.org/10.1017/jfm.2015.257

[78] Lee, J. H. S., The Detonation Phenomenon, 1st ed., Cambridge Univ. Press, New York, 2008, Chaps. 1, 5.

[79] Park, S.-J., Yu, S.-T., Lai, M.-C., Chang, S.-C., and Jorgenson, P. C. E., "Numerical Calculation of Unstable Detonations by the Method of Space-Time Conservation Element and Solution Element," 37th AIAA Aerospace Science Meeting and Exhibit, AIAA Paper 1999-0491, 1999.

https://doi.org/10.2514/6.1999-491

[80] Zhang, Z.-C., Yu, S.-T. J., He, H., and Chang, S.-C., "Direct Calculations of Two- and Three- Dimensional Detonations by an Extended CE/SE Method," 39th AIAA Aerospace Sciences Meeting and Exhibit, AIAA Paper 2001-0476, 2001. https://doi.org/10.2514/6.2001-476

[81] Weng, C., and Gore, J. P., "A Numerical Study of Two- and Three-Dimensional Detonation Dynamics of Pulse Detonation Engine by the CE/SE Method," Acta Mechanica Sinica, Vol. 21, No. 1, 2005, pp. 32-39. https://doi.org/10.1007/s10409-004-0004-8

[82] Shen, H., Liu, K.-X., and Zhang, D.-L., "Three-Dimensional Simulation of Detonation Propagation in a Rectangular Duct by an Improved CE/SE Scheme," Chinese Physics Letters, Vol. 28, No. 12, 2011, pp. $124705-124708$. https://doi.org/10.1088/0256-307X/28/12/124705

[83] Shi, L., Shen, H., Zhang, P., Zhang, D., and Wen, C.-Y., "Assessment of Vibrational Non-Equilibrium Effect on Detonation Cell Size," Combustion Science and Technology, Vol. 189, No. 5, 2017, pp. 841-853. https://doi.org/10.1080/00102202.2016.1260561

[84] Park, C., "Assessment of Two-Temperature Kinetic Model for Ionizing Air," Journal of Thermophysics and Heat Transfer, Vol. 3, No. 3, 1989, pp. 233-244. https://doi.org/10.2514/3.28771

[85] Wang, B., He, H., and Yu, S.-T. J., "Direct Calculation of Wave Implosion for Detonation Initiation," AIAA Journal, Vol. 43, No. 10, 2005, pp. 2157-2169. https://doi.org/10.2514/1.11887

[86] Shen, H., and Parsani, M., "The Role of Multidimensional Instabilities in Direct Initiation of Gaseous Detonations in Free Space," Journal of Fluid Mechanics, Vol. 813, Feb. 2017, pp. R4-1-R4-12. https://doi.org/10.1017/jfm.2017.5

[87] Shi, L., Uy, K. C. K., and Wen, C.-Y., "The Re-Initiation Mechanism of Detonation Diffraction in a Weakly Unstable Gaseous Mixture," Journal of Fluid Mechanics, Vol. 895, July 2020, pp. A24-1-A24-36. https://doi.org/10.1017/jfm.2020.311

[88] Wang, G., Zhang, D., Liu, K., and Wang, J., "An Improved CE/SE Scheme for Numerical Simulation of Gaseous and Two-Phase Detonations," Computers and Fluids, Vol. 39, No. 1, 2010, pp. 168-177. https://doi.org/10.1016/j.compfluid.2009.07.010

[89] Wang, G., Zhu, H., Sun, Q., Zhang, D., and Liu, K., "An Improved CE/ SE Scheme and Its Application to Dilute Gas-Particle Flows," Computer Physics Communications, Vol. 182, No. 8, 2011, pp. 1589-1601. https://doi.org/10.1016/j.cpc.2011.04.004

[90] Dong, H.-F., Hong, T., and Zhang, D.-L., "Application of the CE/SE Method to a Two-Phase Detonation Model in Porous Media," Chinese Physics Letters, Vol. 28, No. 3, 2011, Paper 030203. https://doi.org/10.1088/0256-307X/28/3/030203

[91] Shen, H., Wang, G., Liu, K., and Zhang, D., "Numerical Simulation of Liquid-Fueled Detonations by an Eulerian-Lagrangian Model," International Journal of Nonlinear Sciences and Numerical Simulation, Vol. 13, No. 2, 2012, pp. 177-188. https://doi.org/10.1515/ijnsns-2011-0102

[92] Zhang, Z., Wen, C.-Y., Liu, Y., Zhang, D., and Jiang, Z., "Application of CE/SE Method to Gas-Particle Two-Phase Detonations Under an Eulerian-Lagrangian Framework," Journal of Computational Physics,
Vol. 394, Oct. 2019, pp. 18-40. https://doi.org/10.1016/j.jcp.2019.05.025

[93] Zhang, F., Gerrard, K., and Ripley, R. C., "Reaction Mechanism of Aluminum-Particle-Air Detonation," Journal of Propulsion and Power, Vol. 25, No. 4, 2009, pp. 845-858. https://doi.org/10.2514/1.41707

[94] Wang, X.-Y., Chang, S.-C., and Jorgenson, P. C. E., "Accuracy Study of the Space-Time CE/SE Method for Computational Aeroacoustics Problems Involving Shock Waves," 38th AIAA Aerospace Science Meeting and Exhibit, AIAA Paper 2000-0474, 2000. https://doi.org/10.2514/6.2000-474

[95] Loh, C. Y., Hultgren, L. S., and Chang, S.-C., "Wave Computation in Compressible Flow Using Space-Time Conservation Element and Solution Element Method," AIAA Journal, Vol. 39, No. 5, 2001, pp. 794-801. https://doi.org/10.2514/2.1412

[96] Yen, J. C., Duell, E. G., and Martindale, W., "CAA Using 3-D CESE Method with a Simplified Courant Number Insensitive Scheme," 12th AIAA/CEAS Aeroacoustics Conference, AIAA Paper 2006-2417, 2006. https://doi.org/10.2514/6.2006-2417

[97] Yen, J. C., "Demonstration of a Multi-Dimensional Time-Accurate Local Time Stepping CESE Method," 17th AIAA/CEAS Aeroacoustics Conference, AIAA Paper 2011-2755, 2011. https://doi.org/10.2514/6.2011-2755

[98] Loh, C. Y., and Zaman, K. B. M. Q., "Numerical Investigation of Transonic Resonance with a Convergent-Divergent Nozzle," AIAA Journal, Vol. 40, No. 12, 2002, pp. 2393-2401. https://doi.org/10.2514/2.1607

[99] Loh, C. Y., Himansu, A., and Hultgren, L. S., "A 3-D CE/SE NavierStokes Solver with Unstructured Hexahedral Grid for Computation of Nearfield Jet Screech Noise," 9th AIAA/CEAS Aeroacoustics Conference and Exhibit, AIAA Paper 2003-3207, 2003. https://doi.org/10.2514/6.2003-3207

[100] Kim, C.-K., Yu, S.-T. J., and Zhang, Z.-C., "Cavity Flow in Scramjet Engine by Space-Time Conservation and Solution Element Method," AIAA Journal, Vol. 42, No. 5, 2004, pp. 912-919. https://doi.org/10.2514/1.9017

[101] Cheng, G. C., Olcmen, S. M., Venkatachari, B. S., Brooker, B. T., and Chang, S.-C., "Computational Study of Subsonic and Supersonic Acoustic Cavity Flows Using CESE Method," 2018 AIAA/CEAS Aeroacoustics Conference, AIAA Paper 2018-3594, 2018. https://doi.org/10.2514/6.2018-3594

[102] Wang, J., Liu, K., and Zhang, D., "An Improved CE/SE Scheme for Multi-Material Elastic-Plastic Flows and Its Applications," Computers and Fluids, Vol. 38, No. 3, 2009, pp. 544-551. https://doi.org/10.1016/j.compfluid.2008.04.014

[103] Chen, Q., Wang, J., and Liu, K., "Improved CE/SE Scheme with Particle Level Set Method for Numerical Simulation of Spall Fracture Due to High-Velocity Impact," Journal of Computational Physics, Vol. 229, No. 19, 2010, pp. 7503-7519. https://doi.org/10.1016/j.jcp.2010.06.033

[104] Yu, S.-T. J., Yang, L., Lowe, R. L., and Bechtel, S. E., "Numerical Simulation of Linear and Nonlinear Waves in Hypoelastic Solids by the CESE Method," Wave Motion, Vol. 47, No. 3, 2010, pp. 168-182. https://doi.org/10.1016/j.wavemoti.2009.09.005

[105] Yang, L., Lowe, R. L., Yu, S.-T. J., and Bechtel, S. E., "Numerical Solution by the CESE Method of a First-Order Hyperbolic Form of the Equations of Dynamic Nonlinear Elasticity," Journal of Vibration and Acoustics, Vol. 132, No. 5, 2010, Paper 051003. https://doi.org/10.1115/1.4001499

[106] Chen, Y.-Y., Yang, L., and Yu, S.-T. J., "Simulations of Waves in Elastic Solids of Cubic Symmetry by the Conservation Element and Solution Element Method," Wave Motion, Vol. 48, No. 1, 2011, pp. 39-61. https://doi.org/10.1016/j.wavemoti.2010.07.001

[107] Yang, L., Chen, Y.-Y., and Yu, S.-T. J., "Velocity-Stress Equations for Waves in Solids of Hexagonal Symmetry Solved by the Space-Time CESE Method," Journal of Vibration and Acoustics, Vol. 133, No. 2, 2011, Paper 021001. https://doi.org/10.1115/1.4002170

[108] Lowe, R. L., Lin, P.-H., Yu, S.-T. J., and Bechtel, S. E., "An Eulerian Model for Nonlinear Waves in Elastic Rods, Solved Numerically by the CESE Method," International Journal of Solids and Structures, Vol. 94, Sept. 2016, pp. 179-195. https://doi.org/10.1016/j.ijsolstr.2016.04.016

[109] Zhang, M., Yu, S.-T. J., Lin, S.-C., Chang, S.-C., and Blankson, I., "Solving Magnetohydrodynamic Equations Without Special Treatment for Divergence-Free Magnetic Field," AIAA Journal, Vol. 42, 
No. 12,2004 , pp. $2605-2608$.

https://doi.org/10.2514/1.8512

[110] Zhang, M., Yu, S.-T. J., Lin, S.-C., Chang, S.-C., and Blankson, I., "Solving the MHD Equations by the Space-Time Conservation Element and Solution Element Method," Journal of Computational Physics, Vol. 214, No. 2, 2006, pp. 599-617. https://doi.org/10.1016/j.jcp.2005.10.006

[111] Feng, X., Xiang, C., Zhong, D., Zhou, Y., Yang, L., and Ma, X., "SIPCESE MHD Model of Solar Wind with Adaptive Mesh Refinement of Hexahedral Meshes," Computer Physics Communications, Vol. 185, No. 7, 2014, pp. 1965-1980. https://doi.org/10.1016/j.cpc.2014.03.027

[112] Wang, X.-Y., Chen, C., and Liu, Y., "The Space-Time CE/SE Method for Solving Maxwell's Equations in Time-Domain," Antennas and Propagation Society International Symposium, Vol. 1, IEEE, New York, 2002, pp. 164-167.

[113] Sessions, W. D., and Winans, K. D., "Development of a Self-Consistent Truly Multiphysics Algorithm Based Upon the Courant-Insensitive Space-Time Conservation-Element Solution-Element Method," IEEE Transactions on Plasma Science, Vol. 39, No. 4, 2011, pp. 988-994. https://doi.org/10.1109/TPS.2011.2124470

[114] Molls, T., and Molls, F., "Space-Time Conservation Method Applied to Saint Venant Equations," Journal of Hydraulic Engineering, Vol. 124, No. 5, 1998, pp. 501-508. https://doi.org/10.1061/(ASCE)0733-9429(1998)124:5(501))

[115] Saleem, M. R., Zia, S., Ashraf, W., Ali, I., and Qamar, S., "The Space-Time CESE Scheme for Shallow Water Equations Incorporating Variable Bottom Topography and Horizontal Temperature Gradients," Computers and Mathematics with Applications, Vol. 75, No. 3, 2018, pp. 933-956. https://doi.org/10.1016/j.camwa.2017.10.021

[116] Lim, Y.-I., Chang, S.-C., and Jorgensen, S. B., "A Novel Partial Differential Algebraic Equation (PDAE) Solver: Iterative Space-Time Conservation Element/Solution Element (CE/SE) Method," Computers and Chemical Engineering, Vol. 28, No. 8, 2004, pp. 1309-1324. https://doi.org/10.1016/j.compchemeng.2003.09.016

[117] Yao, C., Tang, S., Lu, Y., Yao, H.-M., and Tade, M. O. "Combination of Space-Time Conservation Element/Solution Element Method and Continuous Prediction Technique for Accelerated Simulation of Simulated Moving Bed Chromatography," Chemical Engineering and Processing: Process Intensification, Vol. 96, Oct. 2015, pp. 54-61. https://doi.org/10.1016/j.cep.2015.07.023

[118] Chou, Y., and Yang, R.-J., "Application of CESE Method to Simulate Non-Fourier Heat Conduction in Finite Medium with Pulse Surface Heating," International Journal of Heat and Mass Transfer, Vol. 51, Nos. 13-14, 2008, pp. 3525-3534. https://doi.org/10.1016/j.ijheatmasstransfer.2007.10.025

[119] Qamar, S., and Ashraf, W., "A Space-Time CE/SE Method for Solving Hyperbolic Heat Conduction Model," International Journal of Computational Methods, Vol. 11, No. 1, 2014, Paper 1350048 https://doi.org/10.1142/S0219876213500485

[120] Noor, S., and Qamar, S., "The Space Time CE/SE Method for Solving One-Dimensional Batch Crystallization Model with Fines Dissolution," Chinese Journal of Chemical Engineering, Vol. 23, No. 2, 2015, pp. 337-341. https://doi.org/10.1016/i.cjche.2014.10.013

[121] Nisar, U. A., Ashraf, W., and Qamar, S., "A Splitting Scheme Based on the Space-Time CE/SE Method for Solving Multi-Dimensional Hydrodynamical Models of Semiconductor Devices," Computer Physics Communications, Vol. 205, Aug. 2016, pp. 69-86. https://doi.org/10.1016/j.cpc.2016.04.013

[122] Rehman, A., Ali, I., and Qamar, S., "An Upwind Space-Time Conservation Element and Solution Element Scheme for Solving Dusty Gas Flow Model," Results in Physics, Vol. 7, Jan. 2017, pp. 3678-3686. https://doi.org/10.1016/j.rinp.2017.09.031

P. Givi Associate Editor 